\title{
Provable Secure and Efficient Digital Rights Management Authentication Scheme Using Smart Card Based on Elliptic Curve Cryptography
}

\author{
Yuanyuan Zhang, ${ }^{1}$ Muhammad Khurram Khan, ${ }^{2}$ Jianhua Chen, ${ }^{1}$ and Debiao He ${ }^{1}$ \\ ${ }^{1}$ School of Mathematics and Statistics, Wuhan University, Wuhan 430072, China \\ ${ }^{2}$ Center of Excellence in Information Assurance, King Saud University, Riyadh 12372, Saudi Arabia \\ Correspondence should be addressed to Muhammad Khurram Khan; mkhurram@ksu.edu.sa
}

Received 30 June 2014; Accepted 28 July 2014

Academic Editor: Jong-Hyuk Park

Copyright ( 2015 Yuanyuan Zhang et al. This is an open access article distributed under the Creative Commons Attribution License, which permits unrestricted use, distribution, and reproduction in any medium, provided the original work is properly cited.

\begin{abstract}
Since the concept of ubiquitous computing is firstly proposed by Mark Weiser, its connotation has been extending and expanding by many scholars. In pervasive computing application environment, many kinds of small devices containing smart cart are used to communicate with others. In 2013, Yang et al. proposed an enhanced authentication scheme using smart card for digital rights management. They demonstrated that their scheme is secure enough. However, Mishra et al. pointed out that Yang et al.'s scheme suffers from the password guessing attack and the denial of service attack. Moreover, they also demonstrated that Yang et al's scheme is not efficient enough when the user inputs an incorrect password. In this paper, we analyze Yang et al's scheme again, and find that their scheme is vulnerable to the session key attack. And, there are some mistakes in their scheme. To surmount the weakness of Yang et al's scheme, we propose a more efficient and provable secure digital rights management authentication scheme using smart card based on elliptic curve cryptography.
\end{abstract}

\section{Introduction}

In 1991, ubiquitous computing was firstly proposed by Mark Weiser, who thought that ubiquitous computing technology could provide users service with a variety of equipment in environment which would be disappeared from the user's consciousness [1]. Later, IBM Corporation scientists also raised the idea in 1999, and they forecasted that pervasive computing can be a way to compute everywhere, anytime, and anywhere [2,3]. Since the computer and internet technology development, multimedia contents (image, document, music, movie, video, etc.) have been greatly enriched all the time, and all of them can be easily redistributed, copied, and downloaded on the internet without authorization. This drawback results in rampant piracy and causes huge revenue to lose to the electronic commerce [4]. As a result, in pervasive computing application environment, the protection of digital publication copyright becomes more and more important. Digital rights management (DRM) technology is developed to overcome the problem [5]. Normally, DRM is only software which usually restricts the usage of the content to protect copy and distributed contents [6-9]. The DRM system manages the procedure of the digital contents including protection, distribution, and authorization. Using DRM technology, intellectual property is respected and protected by data encryption, so it can only be accessed by authorised users without limitless distribution $[10,11]$.

In 2009, the first three-role based DRM implementation scenario authentication scheme using smart card was proposed by Zhang et al. [12]. Then, Yang et al. showed that Zhang et al's scheme was vulnerable to the insider attack and the stolen smart card attack [10]. Due to surmounting the weaknesses of Zhang et al's scheme, Yang et al. proposed an enhanced digital rights management authentication scheme based on smart card. They demonstrated that their scheme could preclude all the weaknesses existing in Zhang et al's 
scheme. Recently, Mishra and Mukhopadhyay cryptanalyzed Yang et al's scheme and found that their scheme cannot resist the password guessing attack and the denial of service attack. Moreover, they also pointed out that Yang et al.s scheme is not efficient enough when the user inputs an incorrect password, and this drawback may cause a denial of service attack [13]. Except for the attacks mentioned by Mishra et al., we find out that Yang et al's scheme does not resist the session key attack. In addition to this, we also discover that there are some mistakes in their scheme.

We proposed a new efficient and provable secure digital rights management authentication scheme using smart card based on elliptic curve cryptography [14-16]. To demonstrate the scheme is provable secure, we introduce a security model AFP05 $[17,18]$ and analyze our scheme in this model. In the following, we will give the proof that our proposed scheme is secure in the AFP05 model. As known to all, one-way hash function is more efficient than the operation of scalar multiplication and pairings [19-21]. Moreover, the pairing operation costs much more than the scalar multiplication operation. The effort of evaluating one pairing operation is approximately three times the effort of evaluating one scalar multiplication operation. So, we cut down some pairings operation of point on elliptic curve and use hash function instead to increase the scheme's efficiency.

The structure of this paper is arranged as follows. In Section 2, we introduce the notations and definitions used in this paper. Section 3 reviews Yang et al's scheme, and Section 4 discusses its weakness analysis. We show the scheme details we propose in Section 5. Section 6 shows a formal security proof of the scheme, while Section 7 demonstrates the security analysis of our proposed scheme. In Section 8, we compare our proposed scheme with Yang et al's and Zhang et al's scheme. Section 9 concludes the paper.

\section{Notations and Definitions}

Let $G_{1}$ be an additive group with an elliptic curve by the generator $P$ and $G_{2}$ a multiplicative cyclic group by the generator $g$. And both of them have the prime order $q$. Let $e$ denote a computable bilinear map $e: G_{1} \times G_{1} \mapsto G_{2}$ satisfying the following three properties $[10,12]$ :

(i) Computability. Given $R, Q \in G_{1}$, there is an efficient algorithm to compute $e(R, Q)$.

(ii) Bilinear. $e(a R, b Q)=e(R, Q)^{a b}$, where $R, Q \in G_{1}$ and $a, b \in Z_{q}^{*}$.

(iii) Nondegenerate. Let $P$ be the generator of $G_{1}, e(P, P) \neq$ $1_{G_{2}}$.

Several commonly used notations and their descriptions are described after the Conclusions Section to facilitate the following references.

\section{Review of Yang et al.'s Scheme}

There are three phases in their scheme; they are, respectively, registration phase, mutual authentication and key agreement phase, and password update phase.

\subsection{Registration Phase}

3.1.1. User's Registration Section. In this part, a user $U$ requests to be a legal user and the server $S$ conducts the next operations.

$U 1\left(U \rightarrow S:\left\{\mathrm{ID}_{U}, \mathrm{PWD}_{U}\right\}\right)$. The user $U$ generates his/her own identity $\mathrm{ID}_{U}$ and password $\mathrm{PW}_{U}$ freely. Then, $U$ chooses a nonce $N_{U}$ randomly and computes

$$
\mathrm{PWD}_{U}=H_{2}\left(\mathrm{PW}_{U} \oplus N_{U}\right) \text {. }
$$

After that, $U$ sends $\left\{\mathrm{ID}_{U}, \mathrm{PW} D_{U}\right\}$ to the server securely.

$U 2\left(S \rightarrow U:\left\{S_{U}, H_{2}(\cdot)\right\}\right)$. After obtaining the message sent by $U$, the server $S$ begins to compute

$$
\begin{aligned}
K_{U} & =H_{2}\left(s H_{1}\left(\mathrm{ID}_{U}\right)\right), \\
S_{U} & =K_{U} \oplus \mathrm{PW} D_{U}, \\
T_{U} & =\mathrm{PW} D_{U} \oplus K_{U} .
\end{aligned}
$$

Then, $S$ stores $\left\{T_{U}\right\}$ in the verification table. Afterward, the server issues a smart card containing $\left\{S_{U}, H_{2}(\cdot)\right\}$ and transmits it to $U$ through a secure channel.

U3. The $N_{U}$ is input into the smart card by the $U$, which contains $\left\{S_{U}, N_{U}, H_{2}(\cdot)\right\}$, finally.

3.1.2. Device's Registration Section. In this section, the device $V$ requests to be authorized by the $S$, and the following steps should be performed together with the server.

$D 1\left(V \rightarrow S:\left\{\operatorname{ID}_{V}\right\}\right)$. The device $V$ transmits its identity $\left\{\operatorname{ID}_{V}\right\}$ to the server $S$ through a secure channel.

$D 2\left(S \rightarrow V:\left\{S_{V}\right\}\right)$. After obtaining the message sent by $V$, the $S$ begins to compute

$$
S_{V}=s H_{1}\left(\mathrm{ID}_{V}\right)
$$

And it is sent to $V$ via a secure channel. Afterward, the device's public key and secret key are $P_{V}=H_{1}\left(\mathrm{ID}_{V}\right)$ and $S_{V}$.

\subsection{Mutual Authentication and Key Agreement Phase}

$M 1\left(U \rightarrow V:\left\{M_{U}\right\}\right)$. After the $U$ inserts his/her smart card into a smart card reader and inputs his/her identity $\mathrm{ID}_{U}$ and password $\mathrm{PW}_{U}$, the smart card randomly chooses a secret number $r_{U}$ and a nonce $N_{U}^{\prime}$. And it computes

$$
\begin{aligned}
\mathrm{PWD}_{U}^{\prime} & =H_{2}\left(\mathrm{PW}_{U} \oplus N_{U}^{\prime}\right), \\
M_{L 1} & =\mathrm{PWD}_{U}^{\prime} \oplus S_{U}, \\
M_{L 2} & =H_{2}\left(S_{U} \oplus \mathrm{PW} D_{U}^{\prime}\right),
\end{aligned}
$$




$$
\begin{aligned}
\mathrm{PWD}_{U} & =H_{2}\left(\mathrm{PW}_{U} \oplus N_{U}\right), \\
K_{U} & =S_{U} \oplus \mathrm{PW} D_{U} .
\end{aligned}
$$

Next $U$ transmits the message $M_{U}=\left\{E_{K_{U}}\left(r_{U}\right), M_{L 1}, M_{L 2}\right\}$ to $V$.

$M 2\left(V \rightarrow S:\left\{\mathrm{ID}_{V}, \mathrm{ID}_{U}, X, Y, M_{U}\right\}\right)$. Upon obtaining the message sent by $U$, the $V$ chooses a number $r_{V}$ randomly and computes

$$
\begin{gathered}
X=r_{V} P, \\
H_{V}=H_{3}\left(\mathrm{ID}_{V}\left\|\mathrm{ID}_{U}\right\| X \| M_{U}\right), \\
Y=r_{V} P_{V}+H_{V} S_{V} .
\end{gathered}
$$

Then, $V$ transmits the message $\left\{\mathrm{ID}_{V}, \mathrm{ID}_{U}, X, Y, M_{U}\right\}$ to $S$.

$M 3\left(S \rightarrow V:\left\{\mathrm{ID}_{S}, \mathrm{ID}_{V}, \mathrm{ID}_{U}, M_{S 1}, M_{S 2}, M_{S 3}, M_{S 4}, M_{S 5}\right.\right.$, $\left.\left.M_{S 6}\right\}\right)$. When the server receives the message, it computes

$$
K_{U}=H_{2}\left(s H_{1}\left(\mathrm{ID}_{U}\right)\right),
$$

$$
\begin{aligned}
& \mathrm{PWD}_{U}=T_{U} \oplus K_{U}, \\
& \mathrm{PWD}_{U}^{\prime}=M_{L 1} \oplus K_{U} \oplus \mathrm{PWD}_{U}
\end{aligned}
$$

and checks whether $M_{L 2}$ is equal to $H_{2}\left(K_{U} \oplus \mathrm{PW} D_{U} \oplus \mathrm{PW} D_{U}^{\prime}\right)$. If this holds, the $S$ will authenticate $U$ and update $T_{U}$ with $T_{U}^{\prime}=\mathrm{PWD}_{U}^{\prime} \oplus K_{U}$. Otherwise, this authentication request is rejected. Then, $S$ computes

$$
\begin{gathered}
r_{U}^{\prime}=D_{K_{U}}\left(E_{K_{U}}\left(r_{U}\right)\right), \\
H_{V}^{\prime}=H_{3}\left(\mathrm{ID}_{V}\left\|\mathrm{ID}_{U}\right\| X \| M_{U}\right)
\end{gathered}
$$

and checks whether

$$
e(P, Y)=e\left(P_{V}, X+H_{V}^{\prime} P_{S}\right) .
$$

If this holds, the device $V$ is authenticated by $S$. Otherwise, this authentication request is rejected. After that, the server generates two random strings $r_{S 1}, r_{S 3}$, and a random number $r_{S 2} . S$ computes

$$
\begin{aligned}
M_{S 1} & =E_{K_{U}}\left(r_{S 1}\right), \\
M_{S 2} & =E_{r_{S 1}}\left(H_{2}\left(\mathrm{ID}_{S}\left\|\mathrm{ID}_{V}\right\| r_{S 1}\right) \oplus r_{U}^{\prime}\right), \\
M_{S 3} & =r_{S 2} P, \\
k_{S V} & =H_{2}\left(r_{V} r_{S 2} P\right), \\
k_{S U} & =H_{2}\left(r_{U}^{\prime} \| r_{S 1}\right), \\
M_{S 5} & =E_{k_{S V}}\left(r_{S 3}\right), \\
M_{S 6} & =E_{k_{S U}}\left(r_{S 3}\right), \\
h_{S} & =H_{3}\left(\mathrm{ID}_{S}\left\|\mathrm{ID}_{V}\right\| \mathrm{ID}_{U}\left\|M_{S 1}\right\| M_{S 2}\left\|M_{S 3}\right\| M_{S 5} \| M_{S 6}\right), \\
M_{S 4} & =r_{S 2}\left(P_{S}+P_{U}\right)+s h_{S} P .
\end{aligned}
$$

At last, the $S$ replies with the message $\left\{\mathrm{ID}_{S}, \mathrm{ID}_{V}, \mathrm{ID}_{U}, M_{S 1}\right.$, $\left.M_{S 2}, M_{S 3}, M_{S 4}, M_{S 5}, M_{S 6}\right\}$ to $V$.

The correctness of (8) is shown as follows:

$$
\begin{aligned}
e(P, Y) & =e\left(P, r_{V} \cdot P_{V}+H_{V} \cdot S_{V}\right) \\
& =e\left(P, r_{V} \cdot P_{V}\right) e\left(P, H_{V} \cdot S_{V}\right) \\
& =e\left(r_{V} \cdot P, P_{V}\right) e\left(s P, H_{V} \cdot P_{V}\right) \\
& =e\left(P_{V}, X\right) e\left(P_{V}, H_{V} \cdot P_{S}\right) \\
& =e\left(P_{V}, X+H_{V} \cdot P_{S}\right) \\
& =e\left(P_{V}, X+H_{V}^{\prime} \cdot P_{S}\right) .
\end{aligned}
$$

$M 4\left(V \rightarrow U:\left\{\mathrm{ID}_{S}, \mathrm{ID}_{V}, M_{S 1}, M_{S 2}, M_{S 6}, E_{r_{33}^{\prime}}\left(r_{V_{1}}\right)\right\}\right)$. After receiving the message from $U, V$ computes

$$
h_{S}^{\prime}=H_{3}\left(\mathrm{ID}_{S}\left\|\mathrm{ID}_{V}\right\| \mathrm{ID}_{U}\left\|M_{S 1}\right\| M_{S 2}\left\|M_{S 3}\right\| M_{S 5} \| M_{S 6}\right)
$$

and checks whether

$$
e\left(P, M_{S 4}\right)=e\left(P_{S}+P_{U}, M_{S 3}\right) e\left(h_{S}^{\prime} P, P_{S}\right) .
$$

If this holds, the server $S$ is authenticated by the device $V$. Otherwise, this authentication procedure fails. Then, $V$ generates a random string $r_{V_{1}}$ and computes

$$
\begin{gathered}
k_{S V}=H_{2}\left(r_{V} M_{S 3}\right), \\
r_{S 3}^{\prime}=D_{k_{S V}}\left(M_{S 5}\right), \\
E_{r_{S 3}^{\prime}}\left(r_{V_{1}}\right) .
\end{gathered}
$$

Finally, $V$ sends the message $\left\{\mathrm{ID}_{S}, \mathrm{ID}_{V}, M_{S 1}, M_{S 2}, M_{S 6}\right.$, $\left.E_{r_{S 3}^{\prime}}\left(r_{V_{1}}\right)\right\}$ to the user $U$.

The correctness of (12) is shown as follows:

$$
\begin{aligned}
e\left(P, M_{S 4}\right) & =e\left(P, r_{S 2}\left(P_{S}+P_{U}\right)+s h_{S} \cdot P\right) \\
& =e\left(P, r_{S 2}\left(P_{S}+P_{U}\right)\right) e\left(P, s h_{S} \cdot P\right) \\
& =e\left(r_{S 2} \cdot P, P_{S}+P_{U}\right) e\left(s P, h_{S} \cdot P\right) \\
& =e\left(P_{S}+P_{U}, M_{S 3}\right) e\left(h_{S} \cdot P, P_{S}\right) \\
& =e\left(P_{S}+P_{U}, M_{S 3}\right) e\left(h_{S}^{\prime} \cdot P, P_{S}\right) .
\end{aligned}
$$

$M 5\left(U \rightarrow V:\left\{E_{r_{s 3}}\left(r_{U 1}\right)\right\}\right)$. The user $U$ computes

$$
\begin{gathered}
r_{S 1}^{\prime}=D_{K_{U}}\left(M_{S 1}\right) \\
S_{U}^{\prime}=S_{U} \oplus \mathrm{PWD}_{U} \oplus \mathrm{PWD}_{U}^{\prime}
\end{gathered}
$$

and checks whether $D_{r_{S 1}^{\prime}}\left(M_{S 2}\right) \oplus r_{U}$ is equal to $\mathrm{H}_{2}\left(\mathrm{ID}_{S}\left\|\mathrm{ID}_{V}\right\| r_{S 1}^{\prime}\right)$. If it holds, the $S$ is authenticated by the $U$. Otherwise, this authentication procedure fails. Then, $U$ updates $N_{U}$ and $S_{U}$ with $N_{U}^{\prime}$ and $S_{U}^{\prime}$, which are saved in 
the smart card. After that, $U$ generates a random string $r_{U 1}$ and computes

$$
\begin{gathered}
k_{S U}=H_{2}\left(r_{U} \| r_{S 1}\right), \\
r_{S 3}=D_{k_{S U}}\left(M_{S 6}\right), \\
r_{V_{1}}=D_{r_{S 3}}\left(E_{r_{S 3}^{\prime}}\left(r_{V_{1}}\right)\right), \\
E_{r_{S 3}}\left(r_{U 1}\right) .
\end{gathered}
$$

Finally, $U$ generates the session key shared with $V$ by computing $k_{U V}=H_{2}\left(r_{U 1} \| r_{V_{1}}\right)$ and sends the message $\left\{E_{r_{S 3}}\left(r_{U 1}\right)\right\}$ back to the device $V$.

M6. After obtaining the message sent by $U$, the device $V$ computes

$$
\begin{gathered}
r_{U 1}=D_{r_{S 3}}\left(E_{r_{S 3}}\left(r_{U 1}\right)\right), \\
k_{U V}=H_{2}\left(r_{U 1} \| r_{V_{1}}\right) .
\end{gathered}
$$

The $k_{U V}$ is the session key between $U$ and device $V$.

3.3. Password Update Phase. When the user requests to change the password $\mathrm{PW}_{U}$ to a new one $\left(\mathrm{PW}_{\text {new }}\right)$, he/she should perform the next procedures.

$P 1\left(U \rightarrow S:\left\{M_{C}\right\}\right)$. After the $U$ inserts the smart card into a smart card reader and inputs his/her identity $\mathrm{ID}_{U}$, old password $\mathrm{PW}_{U}$ and new password $\mathrm{PW}_{\text {new }}$. Then the smart card chooses a secret nonce $N_{U}^{\prime}$ randomly to compute the following results:

$$
\begin{gathered}
\mathrm{PWD}_{U}^{\prime}=H_{2}\left(\mathrm{PW}_{U} \oplus N_{U}^{\prime}\right), \\
M_{C 1}=\mathrm{PWD}_{U}^{\prime} \oplus S_{U}, \\
\mathrm{PWD}_{\text {new }}=H_{2}\left(\mathrm{PW}_{\text {new }} \oplus N_{U}^{\prime}\right), \\
M_{C 2}=H_{2}\left(S_{U} \oplus \mathrm{PW}_{U}^{\prime} \oplus \mathrm{PW} D_{\text {new }}\right), \\
M_{C 3}=\mathrm{PWD}_{\text {new }} \oplus S_{U} .
\end{gathered}
$$

Then, $U$ transmits $M_{C}=\left\{M_{C 1}, M_{C 2}, M_{C 3}\right\}$ to $S$.

P2 $\left(S \rightarrow U:\left\{M_{C 4}\right\}\right)$. Once obtaining the message sent by $U$, the $S$ computes

$$
\begin{gathered}
K_{U}=H_{2}\left(s H_{1}\left(\mathrm{ID}_{U}\right)\right), \\
\mathrm{PWD}_{U}=T_{U} \oplus K_{U}, \\
\mathrm{PWD}_{U}^{\prime}=M_{C 1} \oplus K_{U} \oplus \mathrm{PWD}_{U}, \\
\mathrm{PWD}_{\text {new }}=M_{C 3} \oplus K_{U} \oplus \mathrm{PWD}_{U}, \\
M_{C 4}=H_{2}\left(\mathrm{PWD}_{\text {new }} \oplus K_{U} \oplus \mathrm{PWD}_{U}\right)
\end{gathered}
$$

and checks whether $M_{C 2}$ is equal to $H_{2}\left(K_{U} \oplus \mathrm{PWD}_{U} \oplus\right.$ $\left.\mathrm{PWD}_{U}^{\prime} \oplus \mathrm{PWD}_{\text {new }}\right)$. If it holds, the $S$ will accept the user's request and update the verifier $T_{U}^{\prime}=\mathrm{PWD}_{\text {new }} \oplus K_{U}$. Otherwise, this request procedure is rejected. Then, the server $S$ transmits the message $\left\{M_{C 4}\right\}$ back to the user $U$.

$P 3$. Once the user obtains the message sent by the server, he/she checks whether $M_{C 4}$ is equal to $H_{2}\left(\mathrm{PWD}_{\text {new }} \oplus S_{U}\right)$. If this holds, the user replaces $N_{U}$ and $S_{U}$ with $N_{U}^{\prime}$ and $S_{U}^{\prime}$, where $S_{U}^{\prime}=S_{U} \oplus \mathrm{PWD}_{U} \oplus \mathrm{PWD}_{\text {new }}$.

\section{Cryptanalysis of Yang et al.'s Scheme}

Recently, Mishra and Mukhopadhyay demonstrated Yang et al's scheme is vulnerable to the password guessing attack and the denial of service attack. Moreover, they also pointed that Yang et al.' scheme does not present efficient login and password change phases such that smart card executes the session in case of incorrect input [13]. Based on the attacks and problems mentioned in Mishra et al.s article, we analyze Yang et al's scheme and find out that the scheme does not resist the session key attack. In addition to this, we also discover that there are some mistakes in their scheme. We will introduce our new discoveries in the following.

4.1. Session Key Attack. If an attacker intercepted the message $\left\{\mathrm{ID}_{S}, \mathrm{ID}_{V}, \mathrm{ID}_{U}, M_{S 1}, M_{S 2}, M_{S 3}, M_{S 4}, M_{S 5}, M_{S 6}\right\}$ which was sent from the server to the device and modified some data in it, the user and the device may establish different session key. So the attacker $A$ can realize the session key attack as the following steps.

(1) The attacker $A$ can intercept and capture the message $\left\{\mathrm{ID}_{S}, \mathrm{ID}_{V}, \mathrm{ID}_{U}, M_{S 1}, M_{S 2}, M_{S 3}, M_{S 4}, M_{S 5}, M_{S 6}\right\}$ sent to device by the server. Next, $A$ generates a number $r_{0}$ randomly and computes

$$
\begin{gathered}
M_{S 3_{0}}=r_{0} P \\
h_{S_{0}}=H_{3}\left(\mathrm{ID}_{S}\left\|\mathrm{ID}_{V}\right\| \mathrm{ID}_{U}\left\|M_{S 1}\right\| M_{S 2}\left\|M_{S 3_{0}}\right\| M_{S 5} \| M_{S 6}\right), \\
M_{S 4_{0}}=r_{0}\left(P_{S}+P_{U}\right)+h_{S_{0}} P_{S} .
\end{gathered}
$$

Then, the message $\left\{\mathrm{ID}_{S}, \mathrm{ID}_{V}, \mathrm{ID}_{U}, M_{S 1}, M_{S 2}, M_{S 3_{0}}, M_{S 4_{0}}\right.$, $\left.M_{S 5}, M_{S 6}\right\}$ is sent to the device $V$ by the attacker $A$.

(2) After receiving the message, $V$ computes

$$
h_{S_{0}}^{\prime}=H_{3}\left(\mathrm{ID}_{S}\left\|\mathrm{ID}_{V}\right\| \mathrm{ID}_{U}\left\|M_{S 1}\right\| M_{S 2}\left\|M_{S 3_{0}}\right\| M_{S 5} \| M_{S 6}\right)
$$

and checks the equation

$$
e\left(P, M_{S 4_{0}}\right)=e\left(P_{S}+P_{U}, M_{S 3_{0}}\right) e\left(h_{S_{0}}^{\prime} P, P_{S}\right) \text {. }
$$

Obviously, they are equal. Then, $V$ generates a random string $r_{V_{1}}$ and computes

$$
\begin{gathered}
k_{S V_{0}}=H_{2}\left(r_{V} M_{S 3_{0}}\right), \\
r_{S 3_{0}}=D_{k_{S V_{0}}}\left(M_{S 5}\right), \\
E_{r_{S 3_{0}}}\left(r_{V_{1}}\right) .
\end{gathered}
$$


Finally, $V$ sends the message $\left\{\mathrm{ID}_{S}, \mathrm{ID}_{V}, M_{S 1}, M_{S 2}, M_{S 6}\right.$, $\left.E_{r_{S 3_{0}}}\left(r_{V_{1}}\right)\right\}$ to the user $U$. In this step, the string $r_{S 3_{0}}$ is not equal to the random string $r_{S 3}$ generated by the server.

The correctness of (22) is shown as follows:

$$
\begin{aligned}
e\left(P, M_{S 4_{0}}\right) & =e\left(P, r_{0}\left(P_{S}+P_{U}\right)+h_{S_{0}} \cdot P_{S}\right) \\
& =e\left(P, r_{0}\left(P_{S}+P_{U}\right)\right) e\left(P, h_{S_{0}} \cdot P_{S}\right) \\
& =e\left(r_{0} \cdot P, P_{S}+P_{U}\right) e\left(h_{S_{0}} \cdot P, P_{S}\right) \\
& =e\left(P_{S}+P_{U}, M_{S 3_{0}}\right) e\left(h_{S_{0}}^{\prime} \cdot P, P_{S}\right) .
\end{aligned}
$$

(3) Because $A$ did not modify the message $\left\{\mathrm{ID}_{S}, \mathrm{ID}_{V}, M_{S 1}\right.$, $\left.M_{S 2},\right\}, D_{r_{S 1}^{\prime}}\left(M_{S 2}\right) \oplus r_{U}$ is equal to $H_{2}\left(\operatorname{ID}_{S}\left\|\mathrm{ID}_{V}\right\| r_{S 1}^{\prime}\right)$. Then, $U$ updates $N_{U}$ and $S_{U}$. After that, $U$ generates a random string $r_{U 1}$ and computes

$$
\begin{gathered}
k_{S U}=H_{2}\left(r_{U} \| r_{S 1}\right), \\
r_{S 3}^{\prime}=D_{k_{S U}}\left(M_{S 6}\right), \\
r_{V_{10}}=D_{r_{S 3}^{\prime}}\left(E_{r_{S 3_{0}}}\left(r_{V_{1}}\right)\right), \\
E_{r_{S 3}^{\prime}}\left(r_{U 1}\right) .
\end{gathered}
$$

Finally, $U$ generates the session key $k_{U V}=H_{2}\left(r_{U 1} \| r_{V_{10}}\right)$ shared with the device and sends the message $\left\{E_{r_{S 3}^{\prime}}\left(r_{U 1}\right)\right\}$ back to the device $V$. In this step, the string $r_{S 3}^{\prime}$ is equal to the random string $r_{S 3}$ generated by the server and not equal to the string $r_{\mathrm{S}_{0}}$ computed by the device.

(4) Once obtaining the message sent by the user, the device computes

$$
\begin{aligned}
& r_{U 1_{0}}=D_{r_{S 3_{0}}}\left(E_{r_{S 3}^{\prime}}\left(r_{U 1}\right)\right), \\
& k_{U V_{0}}=H_{2}\left(r_{U 1_{0}} \| r_{V_{1}}\right),
\end{aligned}
$$

$k_{U V_{0}}$ is the session key shared between the user $U$ and the device $V$. Obviously, the session key $k_{U V}$ computed by the user is different from the session key $k_{U V_{0}}$ computed by the device. So Yang et al's scheme suffers from the session key attack.

4.2. Some Mistakes. In mutual authentication and key agreement phase, the identity $\mathrm{ID}_{U}$ has not been sent to the device $V$. But, when the device computes $H_{V}=$ $H_{3}\left(\mathrm{ID}_{V}\left\|\mathrm{ID}_{U}\right\| X \| M_{U}\right)$, it already knows the user's identity. According to the common sense, if the user has not sent identity to the device, the device cannot obtain the user's identity. So there is a mistake in this phase. What is more, this mistake also exists in the password update phase of Yang et al's scheme.

\section{Our Proposed Scheme}

Based on Yang et al's scheme, our protocol also contains four phases: the registration phase, the login phase, the key agreement phase, and the password update phase. Algorithm 1 describes our scheme's registration phase. The login phase and the key agreement phase will be shown in Algorithm 2. At last, we show the password update phase in Algorithm 3. The detail is shown as the following.

5.1. Registration Phase. In our proposed scheme, the registration phase also can be divided into two parts: the user's registration phase and the device's registration phase. Our device's registration phase is the same as the device's registration phase in Yang et al's scheme. We will describe our user's registration phase as follows.

$R 1\left(U \rightarrow S:\left\{\mathrm{ID}_{U}, \mathrm{PWD}_{U}, \mathrm{IDD}_{U}\right\}\right)$. An identity $\mathrm{ID}_{U}$ and password $\mathrm{PW}_{U}$ are chosen by user $U$ freely. Then, $U$ generates a nonce $r_{U}$ randomly and computes

$$
\begin{aligned}
\mathrm{PWD}_{U} & =H_{2}\left(\mathrm{PW}_{U} \oplus r_{U}\right), \\
\mathrm{IDD}_{U} & =H_{2}\left(\mathrm{ID}_{U} \oplus r_{U}\right) .
\end{aligned}
$$

After that, $U$ sends $\left\{\mathrm{ID}_{U}, \mathrm{PWD}_{U}, \mathrm{IDD}_{U}\right\}$ to the server $S$ via a secure channel.

$R 2\left(S \rightarrow U:\left\{S_{U}, P_{U}, M_{U}, P, H_{2}(\cdot), E_{K}(\cdot) / D_{K}(\cdot)\right\}\right)$. After obtaining the message sent by $U, S$ computes

$$
\begin{aligned}
K_{U} & =H_{2}\left(s H_{1}\left(\mathrm{IDD}_{U}\right)\right), \\
Q_{U} & =H_{2}\left(s H_{1}\left(\mathrm{PWD}_{U}\right)\right), \\
S_{U} & =K_{U} \oplus \mathrm{PWD}_{U} \oplus H_{2}\left(\mathrm{ID}_{U}\right), \\
M_{U} & =Q_{U} \oplus \mathrm{ID}_{U}, \\
P_{U} & =H_{2}\left(K_{U} \oplus Q_{U}\right) .
\end{aligned}
$$

Then, the server $U$ issues a smart card containing $\left\{S_{U}\right.$, $\left.P_{U}, M_{U}, P, H_{2}(\cdot), E_{K}(\cdot) / D_{K}(\cdot)\right\}$ and passes it to $U$ securely.

R3. $U$ enters $r_{U}$ into the smart card, so it contains $\left\{r_{U}\right.$, $\left.S_{U}, P_{U}, M_{U}, P, H_{2}(\cdot), E_{K}(\cdot) / D_{K}(\cdot)\right\}$.

5.2. Login Phase. $U$ inserts his/her smart card into a smart card reader and inputs his/her identity $\mathrm{ID}_{U}$ and password $\mathrm{PW}_{U}$. Then the smart card begins to compute

$$
\begin{aligned}
\mathrm{PWD}_{U} & =H_{2}\left(\mathrm{PW}_{U} \oplus r_{U}\right), \\
K_{U}^{\prime} & =S_{U} \oplus \mathrm{PWD}_{U} \oplus H_{2}\left(\mathrm{ID}_{U}\right), \\
\mathrm{Q}_{U}^{\prime} & =M_{U} \oplus \mathrm{ID}_{U}
\end{aligned}
$$

and checks whether $P_{U}$ is equal to $H_{2}\left(K_{U}^{\prime} \oplus Q_{U}^{\prime}\right)$. If this holds, it will authenticate the identity and password of the user. Otherwise, this user's request procedure is rejected. 


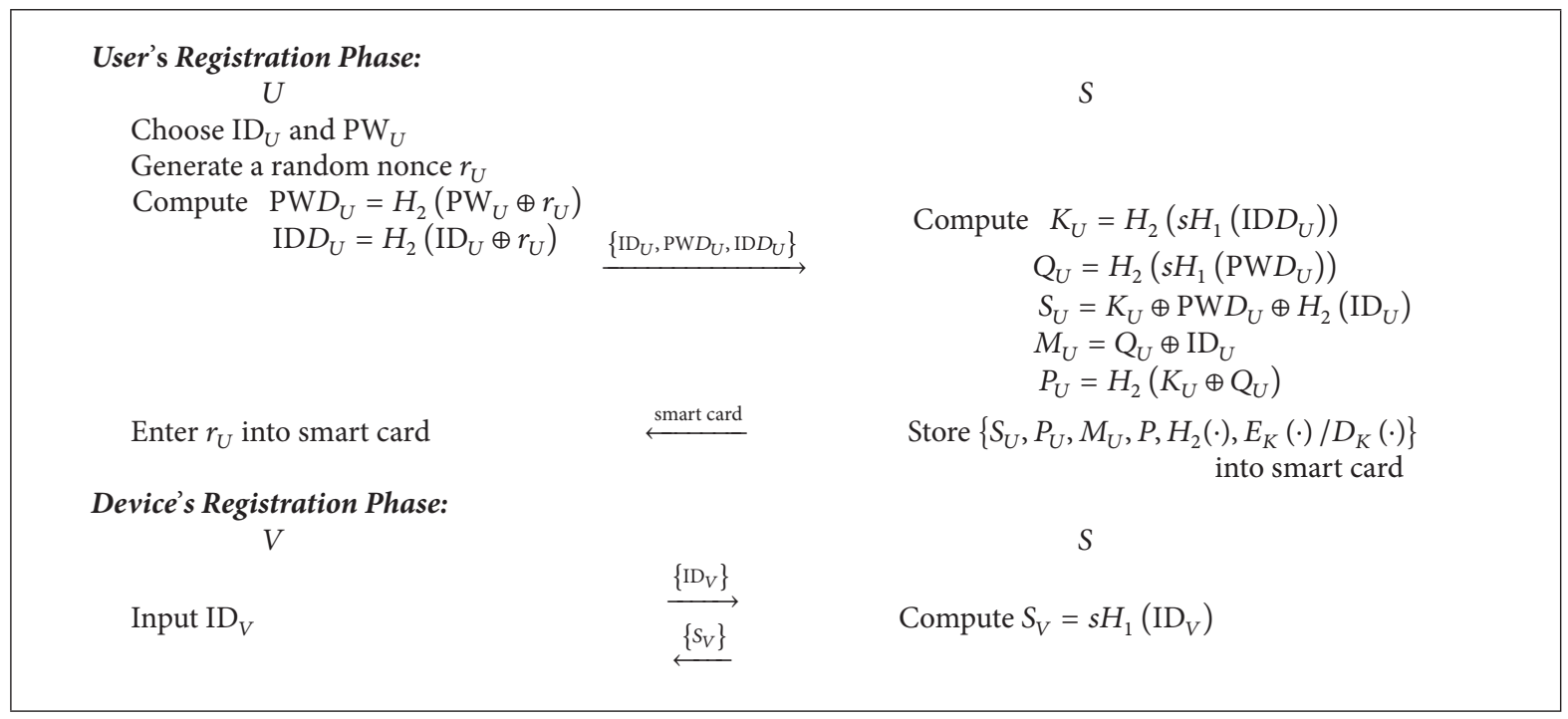

Algorithm 1: The registration phase.

5.3. Key Agreement Phase. There are six steps and five messages during each run of the proposed protocol. The details are as follows.

$A 1\left(U \rightarrow V:\left\{M_{U 2 V_{1}}\right\}\right)$. Then, the smart card generates a secret string $r_{U 1}$ randomly and computes

$$
\begin{gathered}
M_{U 1}=H_{2}\left(K_{U}^{\prime} \oplus Q_{U}^{\prime} \oplus r_{U 1}\right), \\
\mathrm{IDD}_{U}=H_{2}\left(\mathrm{ID}_{U} \oplus r_{U}\right) .
\end{gathered}
$$

Next $U$ transmits the message $M_{U 2 V_{1}}=\left\{\operatorname{IDD}_{U}, E_{K_{U}^{\prime}}\left(r_{U 1}\right)\right.$, $\left.E_{r_{U 1}}\left(\mathrm{PWD}_{U}\right), M_{U 1}\right\}$ to $V$.

$A 2\left(V \rightarrow S:\left\{M_{V_{2} S}\right\}\right)$. Upon obtaining the message sent by $U$, the $V$ generates a number $r_{V_{1}}$ randomly and computes

$$
\begin{gathered}
X=r_{V_{1}} \cdot P \\
H_{V_{1}}=H_{3}\left(\mathrm{ID}_{V}\left\|\mathrm{IDD}_{U}\right\| X \| M_{U 2 V_{1}}\right), \\
Y=r_{V_{1}} \cdot P_{V}+H_{V_{1}} \cdot S_{V} .
\end{gathered}
$$

Then, $V$ transmits the message $M_{V_{2} S}=\left\{\operatorname{ID}_{V}, X, Y, M_{U 2 V_{1}}\right\}$ to the server $S$.

$A 3\left(S \rightarrow V:\left\{M_{S 2 V}\right\}\right)$. When the server received the message, it computes

$$
H_{V_{1}}^{\prime}=H_{3}\left(\mathrm{ID}_{V}\left\|\mathrm{IDD}_{U}\right\| X \| M_{U 2 V_{1}}\right)
$$

and checks whether $e(P, Y)$ is equal to $e\left(P_{V}, X+H_{V_{1}}^{\prime} \cdot P_{S}\right)$. If this holds, the device $V$ is authenticated by $S$. Otherwise, this authentication request is rejected. Then, $S$ computes

$$
\begin{gathered}
K_{U}=H_{2}\left(s H_{1}\left(\operatorname{IDD}_{U}\right)\right), \\
r_{U 1}^{\prime}=D_{K_{U}}\left(E_{K_{U}^{\prime}}\left(r_{U 1}\right)\right), \\
\mathrm{PWD}_{U}^{\prime}=D_{r_{U 1}^{\prime}}\left(E_{r_{U 1}}\left(\mathrm{PWD}_{U}\right)\right), \\
\mathrm{Q}_{U}=H_{2}\left(s H_{1}\left(\mathrm{PWD}_{U}^{\prime}\right)\right)
\end{gathered}
$$

and checks whether $M_{U 1}$ is equal to $H_{2}\left(K_{U} \oplus Q_{U} \oplus r_{U 1}^{\prime}\right)$. If this holds, the user $U$ is authenticated by $S$. Otherwise, this authentication request is rejected. After that, the server $S$ generates a random string $r_{S 1}$. Then $S$ computes

$$
\begin{aligned}
k_{S V} & =s \cdot X=\left(k_{S V x}, k_{S V y}\right), \\
S_{V} & =s \cdot H_{1}\left(\mathrm{ID}_{V}\right)=\left(S_{V x}, S_{V y}\right), \\
M_{S 1} & =k_{S V x} \oplus k_{S V y} \oplus r_{S 1}, \\
M_{S 2} & =H_{2}\left(r_{U 1}^{\prime} \| K_{U}\right) \oplus r_{S 1}, \\
M_{S 3} & =H_{2}\left(r_{U 1}^{\prime} \oplus K_{U} \oplus M_{S 2}\right), \\
M_{S 4} & =H_{2}\left(k_{S V x}\left\|k_{S V y}\right\| S_{V x}\left\|S_{V y}\right\| M_{S 1} \| M_{S 3}\right) .
\end{aligned}
$$

Finally, the $S$ replies with the message $M_{S 2 V}=\left\{M_{S 1}, M_{S 2}\right.$, $\left.M_{S 3}, M_{S 4}\right\}$ to the device $V$.

$A 4\left(V \rightarrow U:\left\{M_{V_{2} U}\right\}\right)$. After receiving the message, $V$ computes

$$
k_{S V}^{\prime}=r_{V_{1}} \cdot P_{S}=\left(k_{S V x}^{\prime}, k_{S V y}^{\prime}\right)
$$




\section{$U$}

\section{Login Phase:}

Input $\mathrm{ID}_{U}$ and $\mathrm{PW}_{U}$

Compute $\mathrm{PWD}_{U}=H_{2}\left(\mathrm{PW}_{U} \oplus r_{U}\right)$

$$
\begin{aligned}
& K_{U}^{\prime}=S_{U} \oplus \mathrm{PWD}_{U} \oplus H_{2}\left(\mathrm{ID}_{U}\right) \\
& Q_{U}^{\prime}=M_{U} \oplus \mathrm{ID}_{U}
\end{aligned}
$$

Check $P_{U} \stackrel{?}{=} H_{2}\left(K_{U}^{\prime} \oplus Q_{U}^{\prime}\right)$

\section{Key agreement Phase:}

Generate a random string $r_{U 1}$

Compute $M_{U 1}=H_{2}\left(K_{U}^{\prime} \oplus Q_{U}^{\prime} \oplus r_{U 1}\right)$

$$
\mathrm{IDD}_{U}=H_{2}\left(\mathrm{ID}_{U} \oplus r_{U}\right)
$$

$M_{U 2 V_{1}}=\left\{\mathrm{IDD}_{U}, E_{K_{U}^{\prime}}\left(r_{U 1}\right), E_{r_{U 1}}\left(\mathrm{PWD}_{U}\right), M_{U 1}\right\}$

$$
\begin{aligned}
& \stackrel{\left\{M_{U 2 V_{1}}\right\}}{\longrightarrow} \text { Generate a random number } r_{V_{1}} \\
& \text { Compute } X=r_{V 1} \cdot P \\
& H_{V_{1}}=H_{3}\left(\mathrm{ID}_{V}\left\|\mathrm{IDD} D_{U}\right\| X \| M_{U 2 V_{1}}\right) \\
& Y=r_{V 1} \cdot P_{V}+H_{V_{1}} \cdot s_{V} \\
& M_{V_{2} S}=\left\{\mathrm{ID}_{V}, X, Y, M_{U 2 V_{1}}\right\} \\
& \stackrel{\left\{M_{V_{2}} S\right\}}{\longrightarrow} \text { Compute } H_{V_{1}}^{\prime}=H_{3}\left(\mathrm{ID}_{V}\left\|\mathrm{IDD}_{U}\right\| X \| M_{U 2 V_{1}}\right) \\
& \text { Check } e(P, Y) \stackrel{?}{=} e\left(P_{V}, X+H_{V_{1}}^{\prime} \cdot P_{S}\right) \\
& \text { Compute } K_{U}=H_{2}\left(s H_{1}\left(\operatorname{IDD}_{U}\right)\right) \\
& r_{U_{1}}^{\prime}=D_{K_{U}}\left(E_{K_{U}^{\prime}}\left(r_{U_{1}}\right)\right) \\
& \mathrm{PWD}_{U}^{\prime}=D_{r_{U 1}^{\prime}}\left(E_{r_{U 1}}\left(\mathrm{PWD}_{U}\right)\right) \\
& Q_{U}=H_{2}\left(s H_{1}\left(\mathrm{PWD}_{U}^{\prime}\right)\right) \\
& \text { Check } \quad M_{U 1} \stackrel{?}{=} H_{2}\left(K_{U} \oplus Q_{U} \oplus r_{U 1}^{\prime}\right)
\end{aligned}
$$

Generate a random string $r_{S 1}$

$$
\begin{array}{cl}
\text { Compute } & k_{S_{V}}=s \cdot X=\left(k_{S_{V} x}, k_{S_{V} y}\right) \\
& S_{V}=s \cdot H_{1}\left(\mathrm{ID}_{V}\right)=\left(S_{V x}, S_{V y}\right) \\
& M_{S 1}=k_{S V x} \oplus k_{S V y} \oplus r_{S 1} \\
& M_{S 2}=H_{2}\left(r_{U 1}^{\prime} \| K_{U}\right) \oplus r_{S 1} \\
& M_{S 3}=H_{2}\left(r_{U 1}^{\prime} \oplus K_{U} \oplus M_{S 2}\right) \\
& M_{S 4}=H_{2}\left(k_{S V x}\left\|k_{S V y}\right\| S_{V x}\left\|S_{V y}\right\| M_{S 1} \| M_{S 3}\right) \\
& M_{S 2 V}=\left\{M_{S 1}, M_{S 2}, M_{S 3}, M_{S 4}\right\}
\end{array}
$$

Compute $k_{S V}^{\prime}=r_{V_{1}} \cdot P_{S}=\left(k_{S V x}^{\prime}, k_{S V y}^{\prime}\right)$

Check $\quad M_{S 4} \stackrel{?}{=} H_{2}\left(k_{S V x}^{\prime}\left\|k_{S V y}^{\prime}\right\| S_{S V x}\left\|S_{S V y}\right\| M_{S 1} \| M_{S 3}\right)$

Generate a random number $r_{V_{2}}$

$$
\begin{array}{cc}
\text { Check } M_{S 3} \stackrel{?}{=} H_{2}\left(r_{U 1} \oplus K_{U}^{\prime} \oplus M_{S 2}\right) \stackrel{\left\{M_{S 2 V}\right\}}{\longleftarrow} \begin{array}{c}
R_{V}=r_{V_{2}} \cdot P=\left(R_{V x}, R_{V y}\right) \\
\text { Compute } r_{S 1}^{\prime \prime}=M_{S 2} \oplus H_{2}\left(r_{U 1} \| K_{U}^{\prime}\right)
\end{array} \quad M_{V_{2} U}=H_{2}\left(R_{V x}\left\|R_{V y}\right\| r_{S 1}^{\prime}\right) \\
\left\{R_{V}, H_{V_{2}}, M_{S 2}, M_{S 3}\right\}
\end{array}
$$$$
\text { Check } H_{V_{2}} \stackrel{?}{=} H_{2}\left(R_{V x}\left\|R_{V y}\right\| r_{S 1}^{\prime \prime}\right)
$$

Generate a random number $r_{U 2}$

Compute $R_{U}=r_{U 2} \cdot P=\left(R_{U x}, R_{U y}\right)$

$$
H_{U}=H_{2}\left(R_{U x}\left\|R_{U y}\right\| r_{S 1}^{\prime \prime}\right)
$$

$$
\begin{array}{llll}
\text { Compute } & R_{U V}=r_{U 2} \cdot R_{V} & \stackrel{\left\{M_{U 2 V_{2}}\right\}}{\longrightarrow} \text { Check } & H_{U} \stackrel{?}{=} H_{2}\left(R_{U x}\left\|R_{U y}\right\| r_{S 1}^{\prime}\right) \\
& k_{U V}=H_{2}\left(R_{U}\left\|R_{V}\right\| R_{U V}\right) & \text { Compute } & R_{U V}^{\prime}=r_{V_{2}} \cdot R_{U} \\
M_{U 2 V_{2}}=\left\{R_{U}, H_{U}\right\} & & k_{U V}^{\prime}=H_{2}\left(R_{U}\left\|R_{V}\right\| R_{U V}^{\prime}\right)
\end{array}
$$




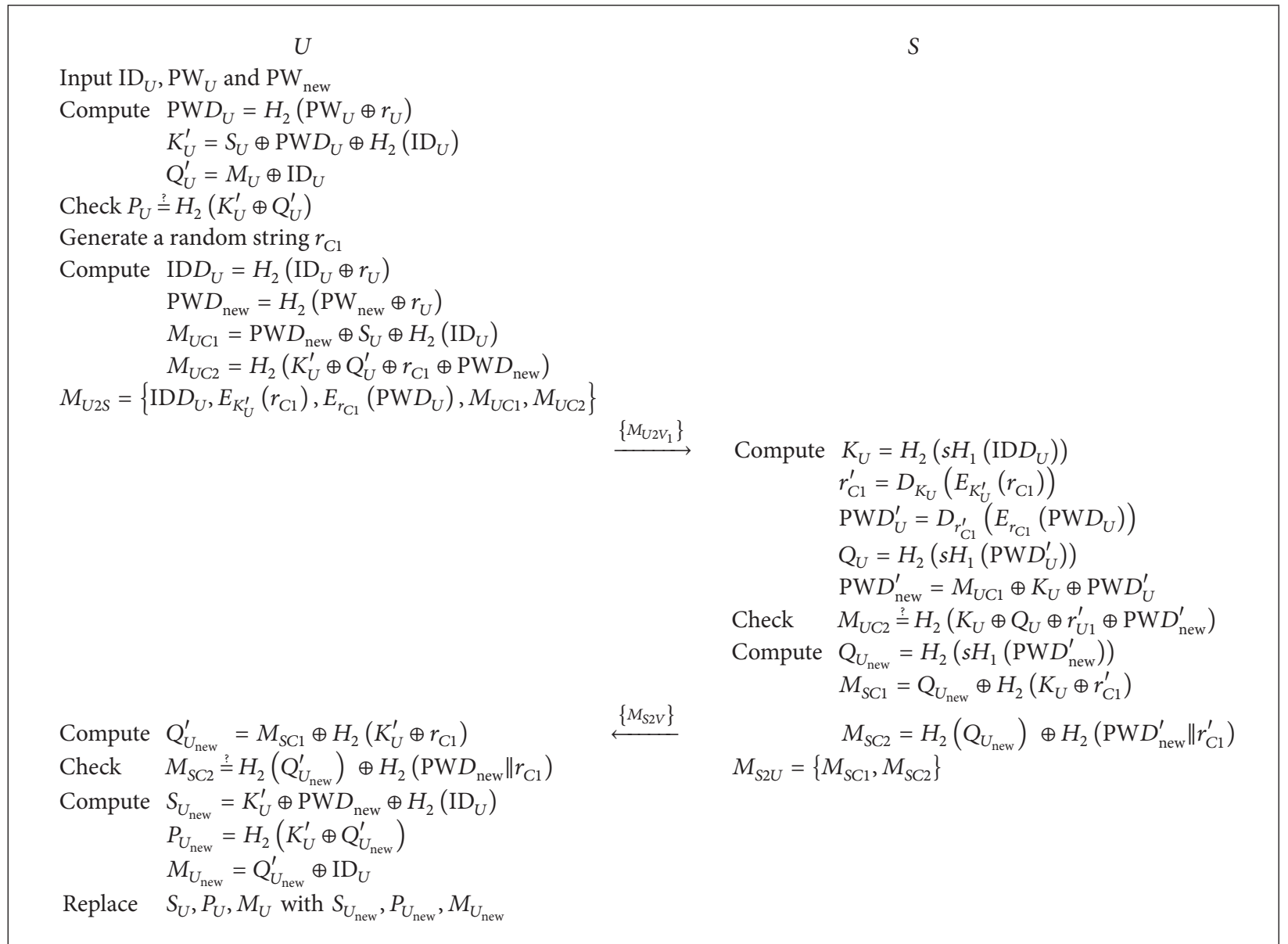

Algorithm 3: The password update phase of our scheme.

and checks whether $M_{S 4}$ is equal to $H_{2}\left(k_{S V x}^{\prime}\left\|k_{S V y}^{\prime}\right\| S_{V x}\left\|S_{V y}\right\| M_{S 1} \| M_{S 3}\right)$. If this holds, the $S$ is authenticated by the device $V$. Otherwise, this authentication procedure fails. Then, $V$ generates a random number $r_{V_{2}}$ and computes

$$
\begin{gathered}
r_{S 1}^{\prime}=M_{S 1} \oplus k_{S V x}^{\prime} \oplus k_{S V y}^{\prime}, \\
R_{V}=r_{V_{2}} \cdot P=\left(R_{V x}, R_{V y}\right), \\
H_{V_{2}}=H_{2}\left(R_{V x}\left\|R_{V y}\right\| r_{S 1}^{\prime}\right) .
\end{gathered}
$$

Finally, the device $V$ transmits the message $M_{V_{2} U}=\left\{R_{V}\right.$, $\left.H_{V_{2}}, M_{S 2}, M_{S 3}\right\}$ to the user $U$.

$A 5\left(U \rightarrow V:\left\{M_{U 2 V_{2}}\right\}\right)$. The user $U$ checks whether $M_{S 3}$ is equal to $H_{2}\left(r_{U 1} \oplus K_{U}^{\prime} \oplus M_{S 2}\right)$. If this holds, the user $U$ authenticates the $S$. Otherwise, this authentication procedure fails. After that, $U$ computes

$$
r_{S 1}^{\prime \prime}=M_{S 2} \oplus H_{2}\left(r_{U 1} \| K_{U}^{\prime}\right) \text {. }
$$

Then, the user $U$ checks whether $H_{V_{2}}$ is equal to $H_{2}\left(R_{V x}\left\|R_{V y}\right\| r_{S 1}^{\prime \prime}\right)$. If this holds, the device $V$ is authenticated by the user $U$. Otherwise, this authentication procedure fails. $U$ generates a random number $r_{U 2}$ and computes

$$
\begin{aligned}
R_{U} & =r_{U 2} \cdot P=\left(R_{U x}, R_{U y}\right), \\
H_{U} & =H_{2}\left(R_{U x}\left\|R_{U y}\right\| r_{S 1}^{\prime \prime}\right), \\
R_{U V} & =r_{U 2} \cdot R_{V}, \\
k_{U V} & =H_{2}\left(R_{U}\left\|R_{V}\right\| R_{U V}\right),
\end{aligned}
$$

where $k_{U V}$ is the session key shared between the user $U$ and the device $V$. Finally, $U$ sends the message $M_{U 2 V_{2}}=\left\{R_{U}, H_{U}\right\}$ back to the device $V$.

A6. After obtaining the message sent by the user, the device $V$ checks whether $H_{U}$ is equal to $H_{2}\left(R_{U x}\left\|R_{U y}\right\| r_{S 1}^{\prime}\right)$. If this holds, the user $U$ is authenticated by the device $V$. Otherwise, this authentication procedure fails. Then, $V$ computes

$$
\begin{aligned}
& R_{U V}^{\prime}=r_{V_{2}} \cdot R_{U}, \\
& k_{U V}^{\prime}=H_{2}\left(R_{U}\left\|R_{V}\right\| R_{U V}^{\prime}\right) .
\end{aligned}
$$

The $k_{U V}^{\prime}$ is the session key between the user $U$ and the device $V$. 
5.4. Password Update Phase. When the user requests to update his/her password $\mathrm{PW}_{U}$ to a new $\mathrm{PW}_{\text {new }}$, he/she should perform the next procedures.

$C 1\left(U \rightarrow S:\left\{\mathrm{IDD}_{U}, E_{K_{U}^{\prime}}\left(r_{C 1}\right), E_{r_{C 1}}\left(\mathrm{PWD}_{U}\right), M_{U C 1}, M_{U C 2}\right\}\right)$. $U$ inserts his/her smart card into a smart card reader and inputs his/her identity $\mathrm{ID}_{U}$, old password $\mathrm{PW}_{U}$, and new password $\mathrm{PW}_{\text {new }}$. Then the smart card checks whether $P_{U}$ is equal to $H_{2}\left(S_{U} \oplus H_{2}\left(\mathrm{PW}_{U} \oplus r_{U}\right) \oplus M_{U} \oplus \mathrm{ID}_{U}\right)$. If this holds, it will authenticate the identity and password of the user. Otherwise, this user's password update request procedure is rejected. After this, the smart card generates a secret string $r_{C 1}$ randomly and computes

$$
\begin{gathered}
K_{U}^{\prime}=S_{U} \oplus H_{2}\left(\mathrm{PW}_{U} \oplus r_{U}\right) \oplus H_{2}\left(\mathrm{ID}_{U}\right), \\
\mathrm{Q}_{U}^{\prime}=M_{U} \oplus \mathrm{ID}_{U}, \\
\mathrm{ID}_{U}=H_{2}\left(\mathrm{ID}_{U} \oplus r_{U}\right), \\
\mathrm{PW} D_{\text {new }}=H_{2}\left(\mathrm{PW}_{\text {new }} \oplus r_{U}\right), \\
M_{U C 1}=\mathrm{PW}_{\text {new }} \oplus S_{U} \oplus H_{2}\left(\mathrm{ID}_{U}\right), \\
M_{U C 2}=H_{2}\left(K_{U}^{\prime} \oplus Q_{U}^{\prime} \oplus r_{C 1} \oplus \mathrm{PWD}_{\text {new }}\right) .
\end{gathered}
$$

Next $U$ transmits the message $\left\{\operatorname{IDD}_{U}, E_{K_{U}^{\prime}}\left(r_{C 1}\right)\right.$, $\left.E_{r_{C 1}}\left(\mathrm{PWD}_{U}\right), M_{U C 1}, M_{U C 2}\right\}$ to the $S$.

$C 2\left(S \rightarrow U:\left\{M_{S C 1}, M_{S C 2}\right\}\right)$. Once obtaining the message sent by the user, the $S$ computes

$$
\begin{gathered}
K_{U}=H_{2}\left(s H_{1}\left(\operatorname{IDD}_{U}\right)\right), \\
r_{C 1}^{\prime}=D_{K_{U}}\left(E_{K_{U}^{\prime}}\left(r_{C 1}\right)\right), \\
\mathrm{PWD}_{U}^{\prime}=D_{r_{C 1}^{\prime}}\left(E_{r_{C 1}}\left(\mathrm{PWD}_{U}\right)\right), \\
Q_{U}=H_{2}\left(s H_{1}\left(\mathrm{PWD}_{U}^{\prime}\right)\right), \\
\mathrm{PWD}_{\text {new }}^{\prime}=M_{U C 1} \oplus K_{U} \oplus \mathrm{PWD}_{U}^{\prime}
\end{gathered}
$$

and checks whether $M_{U C 2}$ is equal to $H_{2}\left(K_{U} \oplus Q_{U}\right) \oplus r_{C 1}^{\prime} \oplus$ PWD $\left.D_{\text {new }}^{\prime}\right)$. If this holds, $S$ will accept the user's request. Otherwise, this request procedure is rejected. Then, the server $S$ computes

$$
\begin{gathered}
Q_{U_{\text {new }}}=H_{2}\left(s H_{1}\left(\mathrm{PWD}_{\text {new }}^{\prime}\right)\right), \\
M_{S C 1}=Q_{U_{\text {new }}} \oplus H_{2}\left(K_{U} \oplus r_{C 1}^{\prime}\right), \\
M_{S C 2}=H_{2}\left(Q_{U_{\text {new }}}\right) \oplus H_{2}\left(P W D_{\text {new }}^{\prime} \| r_{C 1}^{\prime}\right)
\end{gathered}
$$

and sends the message $\left\{M_{S C 1}, M_{S C 2}\right\}$ back to the user $U$.

C3. When the user received the message from the server, he/she computes

$$
Q_{U_{\text {new }}}^{\prime}=M_{S C 1} \oplus H_{2}\left(K_{U}^{\prime} \oplus r_{C 1}\right)
$$

and checks whether $M_{S C 2}$ is equal to $H_{2}\left(Q_{U_{\text {new }}}^{\prime}\right) \oplus$ $\mathrm{H}_{2}\left(\mathrm{PWD} D_{\text {new }} \oplus r_{\mathrm{Cl}}\right)$. If not, the request is rejected. Otherwise, the user computes

$$
\begin{aligned}
S_{U_{\text {new }}} & =K_{U}^{\prime} \oplus \mathrm{PWD}_{\text {new }} \oplus H_{2}\left(\mathrm{ID}_{U}\right), \\
P_{U_{\text {new }}} & =H_{2}\left(K_{U}^{\prime} \oplus Q_{U_{\text {new }}}^{\prime}\right), \\
M_{U_{\text {new }}} & =Q_{U_{\text {new }}}^{\prime} \oplus \mathrm{ID}_{U}
\end{aligned}
$$

and replaces $S_{U}, P_{U}$, and $M_{U}$ with $S_{U_{\text {new }}}, P_{U_{\text {new }}}$, and $M_{U_{\text {new }}}$, which are all saved in the smart card.

\section{Security Model and Proof}

In this part, the provable secure method will be employed to prove that our proposed protocol is provable secure in the models in [18].

6.1. Security Model. In 2005, Abdalla et al. proposed a security model AFP05, which is suitable for the three-party authenticated key agreement scenario. It contains two types of participants, such as the client and the trusted server [18]. But there are three types of participants in our proposed protocol, a user, a trusted device, and a trusted server. So we add a query SendDevice in our security model. During the execution of the protocol, $U$ and $V$ have many instances, respectively. $U_{i}$ and $V_{j}$ denote the $i$ th instance of $U$ and the $j$ th instance of $V$. There exists one state of accept, reject, and $\perp$ in an oracle. If the oracle gets correct message, it turns the accept state; otherwise, it turns reject. $\perp$ means that no decision has been reached or no result has been returned. The adversary $A$, which is abstracted as a probabilistic polynomial time Turing Machine, interacts with other participants through a bounded number of queries which model the capabilities of the adversary in an actual attack. The queries are listed as follows.

SendClient $\left(U_{i}, m\right)$. After receiving message $m$ sent by the adversary, the $U_{i}$ generates a message and outputs as the result of this query. A query SendClient $\left(U_{i}\right.$, Start) begins a new key agreement process.

SendDevice $\left(V_{j}, m\right)$. After receiving message $m$ sent by the adversary, the $V_{j}$ generates a message and output.

SendServer $(S, m)$. After receiving message $m$ sent by the adversary, the $S$ generates a message and output.

Reveal $\left(U_{i} / V_{j}\right)$. If no session key is defined for instance $U_{i} / V_{j}$ or if either $U_{i} / V_{j}$ or its partner is asked a Test query, the result of this query is the invalid symbol $\perp$. Otherwise the session key generated by the instance $U_{i} / V_{j}$ is returned.

$\operatorname{Test}\left(U_{i} / V_{j}\right)$. If no session key is defined for instance $U_{i} / V_{j}$ or if either $U_{i} / V_{j}$ or its partner is asked a Reveal query, the result of this query is the invalid symbol $\perp$. Otherwise, the oracle flips a coin $b$. If $b=1$, the session key is output. Otherwise, a value randomly chosen from the distribution space of session key is output. 
The queries defined in our improved AFP05 security model can be simulated using the SendClient, SendDevice, and SendServer queries repeatedly if we assume that there is at least one benign adversary which faithfully relays message flows. In our improved AFP05 security model, the notion of freshness is already embedded in the definition of the oracles. A Find-Then-Guess (FTG) model exists in our improved AFP05 security model, in which the semantic security is defined by a game with two phases. In the first phase, the adversary is able to adaptively execute SendClient, SendDevice, SendServer, Reveal, and Test query. In the second, $A$ executes a single Test query and guesses a bit $b^{\prime}$ for $b$, where $b$ is selected in the Test query. If $b^{\prime}=b$, the adversary wins the game. Let Succ denote the event that the adversary correctly guesses the bit $b$, and the advantage of $A$ that attacks the protocol $S$ is defined as

$$
\operatorname{Adv}_{S}^{\text {FTG-3PAKA }}(A)=\mid 2 \operatorname{Pr}[\text { Succ }]-1 \mid .
$$

A 3PAKA protocol $S$ is considered semantically secure in FTG model if and only if $\operatorname{Adv}_{S}^{\mathrm{FTG}-3 \mathrm{PAKA}}(t, Q)=$ $\max _{A}\left\{\operatorname{Adv}_{S}^{\mathrm{FTG}-3 \mathrm{PAKA}}(A)\right\}$ is negligible, where the maximum time executed by all the adversaries with time-complexity at most $t$ and the number of queries at most $Q$.

6.2. Mathematical Computational Problems [10]. Let $p, r$ be two large prime numbers and $r \mid(p-1)$. Let $G$ be a multiplicative subgroup of $Z_{p}^{*}$, with prime number $r$ order and element $g_{0}$ generator.

Computational Diffie-Hellman Problem (CDH). Given $\left\{g_{0}, g_{0}^{a}\right.$, $\left.g_{0}^{b} \in G\right\}$ and $a, b \in_{R} Z_{r}^{*}$, it is hard to compute $g^{a b} \bmod p$.

The probabilistic polynomial time Turing Machine denoted as $\Delta$, the probability of which could successfully solve $\mathrm{CDH}$ problem in $G$, is defined as

$$
\operatorname{Succ}_{G}^{\mathrm{CDH}}(\Delta)=\operatorname{Pr}\left[\Delta\left(g^{a}, g^{b}\right)=g^{a b} \bmod p: a, b \in_{R} Z_{r}^{*}\right] .
$$

$C D H$ Assumption. For any probabilistic Turing Machine $\Delta$, the probability of $\operatorname{Succ}_{G}^{\mathrm{CDH}}(\Delta)$ is negligible.

Elliptic Curve Computational Diffie-Hellman (ECCDH) Assumption. Let $G_{1}$ be an additive point group with an elliptic curve by the generator $P$ and $a P$ and $b P$ are elements of $G_{1}$. A is able to compute the value of $a b P$ only with $a P$ and $b P$ in time $t$ at most

$$
\operatorname{Succ}_{G_{1}}^{\mathrm{ECCDH}}(\Delta, t)=\operatorname{Pr}\left[\Delta(a P, b P)=a b P: a, b \in_{R} Z_{q}^{*}\right] .
$$

The fact $\operatorname{Succ}_{G_{1}}^{\mathrm{ECCDH}}(\Delta, t)$ is negligible means that the ECCDH assumption holds.

\subsection{Security Proof}

Theorem 1. The hash functions $H_{1}(\cdot), H_{2}(\cdot)$, and $H_{3}(\cdot)$ are modeled as random oracles. Let $A$ be an adversary against our protocol $S$ within time $t$. We denote that $q_{1}, q_{2}$, and $q_{3}$, respectively, represent the number of $H_{1}(\cdot), H_{2}(\cdot)$, and $H_{3}(\cdot)$ oracle queries executed by $A$, and $q_{s c}, q_{s v}$, and $q_{s s}$ denote the number of SendClient queries, SendDevice queries, and SendServer queries. Then,

$$
\begin{aligned}
\operatorname{Adv}_{S}^{F T G-D R M}(t, Q) \leq & \frac{q_{1}^{2}+q_{3}^{2}+q_{s s}+10 q_{2}+2 q_{3}}{q-1} \\
& +\frac{q_{2}^{2}+2 q_{s s}+4 q_{s c}+4 q_{s v}}{2^{l}} \\
& +2 q_{2} \operatorname{Succ}_{P, G_{1}}^{C D H}\left(t^{\prime}\right),
\end{aligned}
$$

where $Q=\left\{q_{1}, q_{2}, q_{3}, q_{s c}, q_{s v}, q_{s s}\right\}$ and $t^{\prime} \leq t+\left(q_{s c}+q_{s v}+\right.$ $\left.q_{s s}\right) T_{s}$, with $T_{s}$ denoting the computational time of one scalar multiplication in $G_{1}$.

Proof. We define several attack games from Game $G M_{0}$ to Game $G M_{6}$. For each game $G M_{i}$, Succ ${ }_{i}$ denotes the event that $A$ has successfully guessed the bit $b$ in the test session. The games are listed as follows.

Hash, Reveal, Test Queries.

(i) For a hash query $H(i, *, h), i \in\{1,2,3\}$, we proceed as follows:

(a) Rule $H^{(1)}$ : If a record $(i, *, h)$ exists on list $L_{h}, h$ is returned. Otherwise, $h \leftarrow G_{1}(i=1) /\{0,1\}^{l}(i=$ $2) / Z_{q}^{*}(i=3)$ and $h$ is returned. Record $(i, *, h)$ is added to list $L_{h}$. If the adversary directly issues this query, the record $(i, *, h)$ is added to list $L_{A}$.

(ii) For a query Reveal $\left(U_{i} / V_{j}\right)$, we proceed as follows:

(a) If no session key is defined for instance $U_{i} / V_{j}$ or if either $U_{i} / V_{j}$ or its partner is asked a Test query, the output is $\perp$. Otherwise the output of this query is $k_{U V}$ which is defined for the instance $U_{i} / V_{j}$.

(iii) For a query Test $\left(U_{i} / V_{j}\right)$, we proceed as follows:

(a) If no session key is defined for instance $U_{i} / V_{j}$ or if either $U_{i} / V_{j}$ or its partner is asked a Reveal query, the output is $\perp$. Otherwise, the oracle flips a bit $b$. If $b=1$, the session key is output. Otherwise, a value randomly chosen from the distribution space of session key is output.

Game $G M_{0}$. This is the actual attack game. According to the definition, we have

$$
\operatorname{Adv}_{P}^{\text {FTG-DRM }}(A)=\left|2 \operatorname{Pr}\left[\operatorname{Succ}_{0}\right]-1\right| .
$$

Otherwise, we randomly generate a bit $b^{\prime}$ if the game aborts or stops without answer from $A$ or $A$ has not finished the game.

Game $G M_{1}$. We simulate all the oracles for each query and keep three lists to store the oracles answers. $L_{h}$ stores answers 
of random oracles $H_{1}(\cdot), H_{2}(\cdot)$, and $H_{3}(\cdot) . L_{A}$ is denoted for random oracle query asked by $A . L_{S}$ is for the transcripts in the channel. We simulate the SendClient, SendDevice, SendServer, Reveal, and Test queries as in the actual attack. We list hash, Reveal, Test queries in Hash, Reveal, Test Queries and SendClient, SendDevice, SendServer queries in SendClient, SendDevice, SendServer Queries. Obviously, game $G M_{0}$ and game $G M_{1}$ are indistinguishable. So we have

$$
\operatorname{Pr}\left[\mathrm{Succ}_{1}\right]=\operatorname{Pr}\left[\mathrm{Succ}_{0}\right] \text {. }
$$

Game $G M_{2}$. In this game, all the oracles simulated are almost the same as in the game $G M_{1}$, but here, we avoid some collisions in the transcripts. The hash oracles $H_{1}(\cdot), H_{2}(\cdot)$, and $H_{3}(\cdot)$ may collide with different input values. We use the following rule.

Rule $H^{(2)}$. If this query is directly issued by the adversary, and $(i, *, h)_{i \in\{1,2,3\}} \in L_{A}$, the game abort; Otherwise, $h$ is returned.

Given that hash value $h$ is selected from a random uniform distribution, the probability of collisions is at most $\left(q_{1}^{2} / 2(q-1)\right)+\left(q_{2}^{2} / 2^{l+1}\right)+\left(q_{3}^{2} / 2(q-1)\right)[22]$.

Game $G M_{2}$ and game $G M_{1}$ are perfectly indistinguishable unless the abovementioned rule causes the game abort. Hence,

$$
\left|\operatorname{Pr}\left[\mathrm{Succ}_{2}\right]-\operatorname{Pr}\left[\mathrm{Succ}_{1}\right]\right| \leq \frac{q_{1}^{2}}{2(q-1)}+\frac{q_{2}^{2}}{2^{l+1}}+\frac{q_{3}^{2}}{2(q-1)},
$$

Game $G M_{3}$. The game $G M_{3}$ is defined by aborting the executions in which the adversary has obtained a valid authenticator without asking the corresponding hash query by guessing $Y, M_{U 1}, M_{S 4}, M_{S 3}, H_{V_{2}}$, or $H_{U}$. The following rules are used.

Rule $S 1^{(3)}: H_{V_{1}}^{\prime} \leftarrow H_{3}\left(\mathrm{ID}_{V}\left\|\mathrm{IDD}_{U}\right\| X \| M_{U 2 V_{1}}\right)$. We check the equation $e(P, Y)=e\left(P_{V}, X+H_{V_{1}}^{\prime} \cdot P_{S}\right)$. If it does not hold, $S$ terminates without accepting; else we verify whether $\left\{\left(\mathrm{IDD}_{U}, *, *, *\right), \quad\left(\mathrm{ID}_{V}, *, Y, *\right), \quad(*, *, *, *), \quad(*, *, *, *)\right.$, $(*, *)\} \in L_{S}$ or $\left(3,\left(\mathrm{ID}_{V}\left\|\mathrm{IDD}_{U}\right\| X \| M_{U 2 V_{1}}\right), H_{V_{1}}\right) \in L_{A}$. If the two tests fail, $S$ rejects the authenticator and terminates without accepting; otherwise, $S$ accepts and continues.

Rule $S 2^{(3)}: K_{U} \leftarrow H_{2}\left(s H_{1}\left(\mathrm{IDD}_{U}\right)\right), r_{U 1}^{\prime} \leftarrow D_{K_{U}}\left(E_{K_{U}^{\prime}}\left(r_{U 1}\right)\right)$, $\mathrm{PWD}_{U}^{\prime} \leftarrow D_{r_{U 1}^{\prime}}\left(E_{r_{U 1}}\left(\mathrm{PWD}_{U}\right)\right), \mathrm{Q}_{U} \leftarrow H_{2}\left(s H_{1}\left(\mathrm{PWD}_{U}^{\prime}\right)\right)$. We check the equation $H_{2}\left(K_{U} \oplus Q_{U} \oplus r_{U 1}^{\prime}\right)=M_{U 1}$. If it does not hold, $S$ terminates without accepting; else we verify whether $\left(2,\left(K_{U} \oplus Q_{U} \oplus r_{U 1}^{\prime}\right), M_{U 1}\right) \in L_{A}$ or $\left\{\left(\operatorname{IDD}_{U}, *, *, M_{U 1}\right)\right.$, $\left.\left(\operatorname{ID}_{V}, *, *, *\right),(*, *, *, *),(*, *, *, *),(*, *)\right\} \in L_{S}$. If the two tests fail, $S$ rejects the authenticator and terminates without accepting; Otherwise, $S$ accepts and continues.

SendClient, SendDevice, SendServer Queries.

(i) For a query SendClient $\left(U_{i}\right.$, Start), we proceed as follows:

(a) Rule $U 1^{(1)}: r_{U 1} \leftarrow\{0,1\}^{l}, M_{U 1} \leftarrow H_{2}\left(K_{U}^{\prime} \oplus\right.$
$\left.Q_{U}^{\prime} \oplus r_{U 1}\right), \mathrm{IDD}_{U} \leftarrow H_{2}\left(\mathrm{ID}_{U} \oplus r_{U}\right)$ and
$M_{U 2 V_{1}}=\left\{\mathrm{IDD}_{U}, E_{K_{U}^{\prime}}\left(r_{U 1}\right), E_{r_{U 1}}\left(\mathrm{PWD}_{U}\right), M_{U 1}\right\}$ is returned. Then, instance $U_{i}$ proceeds to an expecting state.

(ii) For a query SendClient $\left(U_{i}, M_{V_{2} U}\right)$, we proceed as follows if instance $U_{i}$ is in an expecting state:

(a) Rule $U 2^{(1)}$ : We check whether $H_{2}\left(r_{U 1} \oplus K_{U}^{\prime} \oplus\right.$ $\left.M_{S 2}\right)=M_{S 3}$. If the equation does not hold, instance $U_{i}$ terminates without accepting. Otherwise, instance $U_{i}$ accepts and applies the following rule.

(b) Rule $U 3^{(1)}: r_{S 1}^{\prime \prime} \leftarrow M_{S 2} \oplus H_{2}\left(r_{U 1} \| K_{U}^{\prime}\right)$, and we check whether $H_{2}\left(R_{V x}\left\|R_{V y}\right\| r_{S 1}^{\prime \prime}\right)=H_{V_{2}}$. If the equation does not hold, instance $U_{i}$ terminates without accepting. Otherwise, instance $U_{i}$ accepts and applies the following rule.

(c) Rule $U 4^{(1)}: r_{U 2} \leftarrow Z_{q}^{*}, R_{U} \leftarrow r_{U 2} \cdot P, H_{U} \leftarrow$ $H_{2}\left(R_{U x}\left\|R_{U y}\right\| r_{S 1}^{\prime \prime}\right)$, and instance $U_{i}$ accepts and applies the following rule.

(d) Rule $U 5^{(1)}: R_{U V} \leftarrow r_{U 2} \cdot R_{V}, k_{U V} \leftarrow$ $H_{2}\left(R_{U}\left\|R_{V}\right\| R_{U V}\right)$, and $M_{U 2 V_{2}}=\left\{R_{U}, H_{U}\right\}$ is returned. Then, instance $U_{i}$ terminates and record $\left\{\left(\mathrm{IDD}_{U}, E_{K_{U}^{\prime}}\left(r_{U 1}\right), E_{r_{U 1}}\left(\mathrm{PWD}_{U}\right), M_{U 1}\right)\right.$, $\left(\mathrm{ID}_{V}, X, Y, M_{U 2 V_{1}}\right),\left(M_{S 1}, M_{S 2}, M_{S 3}, M_{S 4}\right)$, $\left.\left(R_{V}, H_{V_{2}}, M_{S 2}, M_{S 3}\right),\left(R_{U}, H_{U}\right)\right\}$ is added to list $L_{S}$.

(iii) For a query SendDevice $\left(V_{j}, M_{U 2 V_{1}}\right)$, we proceed as follows:

(a) Rule $V 1^{(1)}: r_{V_{1}} \leftarrow Z_{q}^{*}, X \leftarrow r_{V_{1}} \cdot P, H_{V_{1}} \leftarrow$ $H_{3}\left(\mathrm{ID}_{V}\left\|\mathrm{IDD} D_{U}\right\| X \| M_{U 2 V_{1}}\right), Y \leftarrow r_{V_{1}} \cdot P_{V}+H_{V_{1}}$. $S_{V}$, and $M_{V_{2} S}=\left\{\mathrm{ID}_{V}, X, Y, M_{U 2 V_{1}}\right\}$ is returned. Then, instance $V_{j}$ proceeds to an expecting state.

(iv) For a query SendDevice $\left(V_{j}, M_{S 2 V}\right)$, we proceed as follows if instance $V_{j}$ is in an expecting state:

(a) Rule $V 2^{(1)}: k_{S V}^{\prime} \leftarrow r_{V 1} \cdot P_{S}=\left(k_{S V x}^{\prime}, k_{S V y}^{\prime}\right)$, and we check whether $M_{S 4}=$ $H_{2}\left(k_{S V x}^{\prime}\left\|k_{S V y}^{\prime}\right\| S_{V x}\left\|S_{V y}\right\| M_{S 1} \| M_{S 3}\right)$. If the equation does not hold, instance $V_{j}$ terminates without accepting. Otherwise, instance $V_{j}$ accepts and applies the following rule.

(b) Rule $V 3^{(1)}: r_{V_{2}} \leftarrow Z_{q}^{*}, r_{S 1}^{\prime} \leftarrow M_{S 1} \oplus k_{S V x}^{\prime} \oplus k_{S V y}^{\prime}$, $R_{V} \leftarrow r_{V_{2}} \cdot P, H_{V_{2}} \leftarrow H_{2}\left(R_{V x}\left\|R_{V y}\right\| r_{S 1}^{\prime}\right)$, and $M_{V_{2} U}=\left\{R_{V}, H_{V_{2}}, M_{S 2}, M_{S 3}\right\}$ is returned. Then, instance $V_{j}$ proceeds to an expecting state.

(v) For a query SendDevice $\left(V_{j}, M_{U 2 V_{2}}\right)$, we proceed as follows if instance $V_{j}$ is in an expecting state:

(a) Rule $V 4^{(1)}:$ We check whether $H_{2}\left(R_{U x}\left\|R_{U y}\right\| r_{S 1}^{\prime}\right)=H_{U}$. If the equation does not hold, instance $V_{j}$ terminates without accepting. Otherwise, instance $V_{j}$ accepts and applies the following rule. 
(b) Rule $V 5^{(1)}: R_{U V}^{\prime} \leftarrow r_{V_{2}} \cdot R_{U}, k_{U V}^{\prime} \leftarrow$ $H_{2}\left(R_{U}\left\|R_{V}\right\| R_{U V}^{\prime}\right)$, and instance $V_{j}$ terminates.

(vi) For a query SendServer $\left(S, M_{V_{2} S}\right)$, we proceed as follows:

(a) Rule $S 1^{(1)}: H_{V_{1}}^{\prime} \leftarrow H_{3}\left(\operatorname{ID}_{V}\left\|\operatorname{IDD} D_{U}\right\| X \| M_{U 2 V_{1}}\right)$, and we check whether $e(P, Y)=e\left(P_{V}, X+H_{V_{1}}^{\prime}\right.$. $\left.P_{S}\right)$. If the equation does not hold, instance $S$ terminates without accepting. Otherwise, instance $S$ accepts and applies the following rule.

(b) Rule $S 2^{(1)}: K_{U} \leftarrow H_{2}\left(s H_{1}\left(\operatorname{IDD}_{U}\right)\right), r_{U 1}^{\prime} \leftarrow$ $D_{K_{U}}\left(E_{K_{U}^{\prime}}\left(r_{U 1}\right)\right), \mathrm{PWD}_{U}^{\prime} \leftarrow D_{r_{U 1}^{\prime}}\left(E_{r_{U 1}}\left(\mathrm{PWD}_{U}\right)\right)$, $\mathrm{Q}_{U} \leftarrow \mathrm{H}_{2}\left(s H_{1}\left(\mathrm{PWD}_{U}^{\prime}\right)\right)$ and we check whether $H_{2}\left(K_{U} \oplus Q_{U} \oplus r_{U 1}^{\prime}\right)=M_{U 1}$. If the equation does not hold, instance $S$ terminates without accepting. Otherwise, instance $S$ accepts and applies the following rule.

(c) Rule $S 3^{(1)}: r_{S 1} \leftarrow\{0,1\}^{l}, k_{S V} \leftarrow s \cdot X, S_{V} \leftarrow$ $s \cdot H_{1}\left(\mathrm{ID}_{V}\right), M_{S 1} \leftarrow k_{S V x} \oplus k_{S V y} \oplus r_{S 1}, M_{S 2} \leftarrow$ $H_{2}\left(r_{U 1}^{\prime} \| K_{U}\right) \oplus r_{S 1}, M_{S 3} \leftarrow H_{2}\left(r_{U 1}^{\prime} \oplus K_{U} \oplus M_{S 2}\right)$, $M_{S 4} \leftarrow H_{2}\left(k_{S V x}\left\|k_{S V y}\right\| S_{V x}\left\|S_{V y}\right\| M_{S 1} \| M_{S 3}\right)$, and $M_{S 2 V}=\left\{M_{S 1}, M_{S 2}, M_{S 3}, M_{S 4}\right\}$ is returned. Then, instance $S$ terminates.

Rule $V 2^{(3)}: k_{S V}^{\prime} \leftarrow r_{V_{1}} \cdot P_{S}=\left(k_{S V x}^{\prime}, k_{S V y}^{\prime}\right)$. We check the equation $M_{S 4}=H_{2}\left(k_{S V x}^{\prime}\left\|k_{S V y}^{\prime}\right\| S_{V x}\left\|S_{V y}\right\| M_{S 1} \| M_{S 3}\right)$. If it does not hold, $V_{j}$ terminates without accepting; else we verify whether $\left(2,\left(k_{S V x}^{\prime}\left\|k_{S V y}^{\prime}\right\| S_{V x}\left\|S_{V y}\right\| M_{S 1} \| M_{S 3}\right), M_{S 4}\right) \in L_{A}$ or $\left\{\left(\operatorname{IDD}_{U}, *, *, *\right),\left(\operatorname{ID}_{V}, *, *, *\right),\left(*, *, *, M_{S 4}\right),(*, *, *, *)\right.$, $(*, *)\} \in L_{S}$. If the two tests fail, $V_{j}$ rejects the authenticator and terminates without accepting; otherwise, $V_{j}$ accepts and continues.

Rule $U 2^{(3)}$. We check the equation $H_{2}\left(r_{U 1} \oplus K_{U}^{\prime} \oplus M_{S 2}\right)=$ $M_{S 3}$. If it does not hold, $U_{i}$ terminates without accepting; else we verify whether $\left\{\left(\operatorname{IDD}_{U}, *, *, *\right),\left(\operatorname{ID}_{V}, *, *, *\right)\right.$, $\left.\left(*, *, M_{S 3}, *\right),\left(*, *, M_{S 2}, *\right),(*, *)\right\} \in L_{S}$ or $\left(2,\left(r_{U 1} \oplus K_{U}^{\prime} \oplus\right.\right.$ $\left.\left.M_{S 2}\right), M_{S 3}\right) \in L_{A}$. If the two tests fail, $U_{i}$ rejects the authenticator and terminates without accepting; otherwise, $U_{i}$ accepts and continues.

Rule $U 3^{(3)}: r_{S 1}^{\prime \prime} \leftarrow M_{S 2} \oplus H_{2}\left(r_{U 1} \| K_{U}^{\prime}\right)$. We check the equation $H_{2}\left(R_{V x}\left\|R_{V y}\right\| r_{S 1}^{\prime \prime}\right)=H_{V_{2}}$. If it does not hold, $U_{i}$ terminates without accepting; else we verify whether $\left\{\left(\operatorname{IDD}_{U}, *, *, *\right),\left(\operatorname{ID}_{V}, *, *, *\right),(*, *, *, *),\left(*, H_{V_{2}}, *, *\right)\right.$,

$(*, *)\} \in L_{S}$ or $\left(2,\left(R_{V x}\left\|R_{V y}\right\| r_{S 1}^{\prime \prime}\right), H_{V 2}\right) \in L_{A}$. If the two tests fail, $U_{i}$ rejects the authenticator and terminates without accepting; otherwise, $U_{i}$ accepts and continues.

Rule $V 4^{(3)}$. We check the equation $H_{2}\left(R_{U x}\left\|R_{U y}\right\| r_{S 1}^{\prime}\right)=$ $H_{U}$. If it does not hold, $V_{j}$ terminates without accepting; else we verify whether $\left\{\left(\operatorname{IDD}_{U}, *, *, *\right)\right.$, $\left.\left(\operatorname{ID}_{V}, *, *, *\right),(*, *, *, *),(*, *, *, *),\left(*, H_{U}\right)\right\} \quad \in \quad L_{S}$ or $\left(2,\left(R_{U x}\left\|R_{U y}\right\| r_{S 1}^{\prime}\right), H_{U}\right) \in L_{A}$. If the two tests fail, $V_{j}$ rejects the authenticator and terminates without accepting; otherwise, instance $V_{j}$ accepts and continues.

Game $G M_{3}$ and Game $G M_{2}$ are almost indistinguishable only if the rules mentioned above cause the instance to reject a valid authenticator. Because the adversary deduces the authenticator without issuing the corresponding hash queries, the probability of guessing is at most $\left(q_{s s} / 2(q-1)\right)+$ $\left(q_{s s} / 2^{l}\right)+\left(q_{s v} / 2^{l}\right)+\left(q_{s c} / 2^{l}\right)+\left(q_{s c} / 2^{l}\right)+\left(q_{s v} / 2^{l}\right)$. Hence,

$$
\left|\operatorname{Pr}\left[\mathrm{Succ}_{3}\right]-\operatorname{Pr}\left[\mathrm{Succ}_{2}\right]\right| \leq \frac{q_{s s}+2 q_{s c}+2 q_{s v}}{2^{l}}+\frac{q_{s s}}{2(q-1)} .
$$

Game $G M_{4}$. We define game $G M_{4}$ by aborting the executions in which the adversary may have obtained valid authenticator $Y, M_{U 1}, M_{S 4}, M_{S 3}, H_{V_{2}}$, or $H_{U}$ by guessing the corresponding secret messages and querying the corresponding hash function. We use the following rules.

Rule $S 1^{(4)}: H_{V_{1}}^{\prime} \leftarrow H_{3}\left(\operatorname{ID}_{V}\left\|\operatorname{IDD}_{U}\right\| X \| M_{U 2 V_{1}}\right)$. We check the equation $e(P, Y)=e\left(P_{V}, X+H_{V_{1}}^{\prime} \cdot P_{S}\right)$. If it does not hold, $S$ terminates without accepting; else we confirm whether $\left\{\left(\operatorname{IDD}_{U}, *, *, *\right),\left(\operatorname{ID}_{V}, *, Y, *\right),(*, *, *, *),(*, *, *, *)\right.$,

$(*, *)\} \in L_{S}$. If the test fails, $S$ rejects the authenticator and terminates without accepting; otherwise, we check whether $\left(3,\left(\mathrm{ID}_{V}\left\|\mathrm{IDD} D_{U}\right\| X \| M_{U 2 V_{1}}\right), H_{V_{1}}\right) \in L_{A}$. If this is the case, we abort the game.

Rule $S 2^{(4)}: \quad K_{U} \leftarrow H_{2}\left(s H_{1}\left(\operatorname{IDD}_{U}\right)\right), \quad r_{U 1}^{\prime} \leftarrow$ $D_{K_{U}}\left(E_{K_{U}^{\prime}}\left(r_{U 1}\right)\right), \quad \mathrm{PWD}_{U}^{\prime} \leftarrow D_{r_{U 1}^{\prime}}\left(E_{r_{U 1}}\left(\mathrm{PWD}_{U}\right)\right)$, $Q_{U} \leftarrow H_{2}\left(s H_{1}\left(\mathrm{PWD}_{U}^{\prime}\right)\right)$. We check the equation $H_{2}\left(K_{U} \oplus Q_{U} \oplus r_{U 1}^{\prime}\right)=M_{U 1}$. If it does not hold, $S$ terminates without accepting; else we confirm whether $\left\{\left(\operatorname{IDD}_{U}, *, *, M_{U 1}\right),\left(\operatorname{ID}_{V}, *, *, *\right),(*, *, *, *),(*, *, *, *)\right.$, $(*, *)\} \in L_{S}$. If the test fails, $S$ rejects the authenticator and terminates without accepting; otherwise, we check whether $\left(2,\left(K_{U} \oplus Q_{U} \oplus r_{U 1}^{\prime}\right), M_{U 1}\right) \in L_{A}$. If this is the case, we abort the game.

Rule $V 2^{(4)}: k_{S V}^{\prime} \leftarrow r_{V_{1}} \cdot P_{S}=\left(k_{S V x}^{\prime}, k_{S V y}^{\prime}\right)$. We check the equation $M_{S 4}=H_{2}\left(k_{S V x}^{\prime}\left\|k_{S V y}^{\prime}\right\| S_{V x}\left\|S_{V y}\right\| M_{S 1} \| M_{S 3}\right)$. If it does not hold, $V_{j}$ terminates without accepting; else we confirm whether $\left\{\left(\operatorname{IDD}_{U}, *, *, *\right),\left(\operatorname{ID}_{V}, *, *, *\right),\left(*, *, *, M_{S 4}\right)\right.$, $(*, *, *, *),(*, *)\} \in L_{S}$. If the test fails, $V_{j}$ rejects the authenticator and terminates without accepting; otherwise, we check whether $\left(2,\left(k_{S V x}^{\prime}\left\|k_{S V y}^{\prime}\right\| S_{V x}\left\|S_{V y}\right\| M_{S 1} \| M_{S 3}\right), M_{S 4}\right) \epsilon$ $L_{A}$. If this is the case, we abort the game.

Rule $U 2^{(4)}$. We check the equation $H_{2}\left(r_{U 1} \oplus K_{U}^{\prime} \oplus M_{S 2}\right)=$ $M_{S 3}$. If it does not hold, $U_{i}$ terminates without accepting; else we confirm whether $\left\{\left(\operatorname{IDD}_{U}, *, *, *\right)\right.$, $\left.\left(\mathrm{ID}_{V}, *, *, *\right),\left(*, *, M_{S 3}, *\right),\left(*, *, M_{S 2}, *\right),(*, *)\right\} \in L_{S}$. If the test fails, $U_{i}$ rejects the authenticator and terminates without accepting; otherwise, we check whether 
$\left(2,\left(r_{U 1} \oplus K_{U}^{\prime} \oplus M_{S 2}\right), M_{S 3}\right) \in L_{A}$. If this is the case, we abort the game.

Rule $U 3^{(4)}: r_{S 1}^{\prime \prime} \leftarrow M_{S 2} \oplus H_{2}\left(r_{U 1} \| K_{U}^{\prime}\right)$. We check the equation $H_{2}\left(R_{V x}\left\|R_{V y}\right\| r_{S 1}^{\prime \prime}\right)=H_{V_{2}}$. If it does not hold, $U_{i}$ terminates without accepting; else we confirm whether $\left\{\left(\operatorname{IDD}_{U}, *, *, *\right),\left(\operatorname{ID}_{V}, *, *, *\right),(*, *, *, *),\left(*, H_{V_{2}}, *, *\right)\right.$, $(*, *)\} \in L_{S}$. If the test fails, $U_{i}$ rejects the authenticator and terminates without accepting; otherwise, we check whether $\left(2,\left(R_{V x}\left\|R_{V y}\right\| r_{S 1}^{\prime \prime}\right), H_{V_{2}}\right) \in L_{A}$. If this is the case, we abort the game.

Rule $V 4^{(3)}$. We check the equation $H_{2}\left(R_{U x}\left\|R_{U y}\right\| r_{S 1}^{\prime}\right) \stackrel{?}{=} H_{U}$. If it does not hold, $V_{j}$ terminates without accepting; else we confirm whether $\left\{\left(\operatorname{IDD}_{U}, *, *, *\right),\left(\operatorname{ID}_{V}, *, *, *\right)\right.$, $\left.(*, *, *, *),(*, *, *, *),\left(*, H_{U}\right)\right\} \in L_{S}$. If the test fails, $V_{j}$ rejects the authenticator and terminates without accepting; otherwise, we check whether $\left(2,\left(R_{U x}\left\|R_{U y}\right\| r_{S 1}^{\prime}\right), H_{U}\right) \in L_{A}$. If this is the case, we abort the game.

Games $G M_{4}$ and $G M_{3}$ are almost indistinguishable only if the rules mentioned above cause the game to abort. Because the secret messages corresponding to valid authenticators $M_{U 1}, Y, M_{S 4}, M_{S 2}, H_{V_{2}}$, and $H_{U}$ are deduced by the adversary, the probability of guessing is at most $\left(q_{3}+q_{2}+q_{2}+q_{2}+q_{2}+\right.$ $\left.q_{2}\right) /(q-1)$. Hence,

$$
\left|\operatorname{Pr}\left[\mathrm{Succ}_{4}\right]-\operatorname{Pr}\left[\mathrm{Succ}_{3}\right]\right| \leq \frac{5 q_{2}+q_{3}}{q-1}
$$

Game $G M_{5}$. Here we simulate a private hash function $H_{4}(\cdot)$ : $\{0,1\}^{*} \mapsto\{0,1\}^{l}$ and take place of random oracle $H_{2}(\cdot)$ with $H_{4}(\cdot)$. Then, we do not use the $R_{U V}$ or $R_{U V}^{\prime}$ to generate session key $k_{U V}$ or $k_{U V}^{\prime}$. As the result, the session key is completely independent of $H_{2}(\cdot)$ and either $R_{U V}$ or $R_{U V}^{\prime}$.

Rule $U 5^{(5)}: R_{U V} \leftarrow r_{U 2} \cdot R_{V}, k_{U V} \leftarrow H_{2}\left(R_{U} \| R_{V}\right)$, and $M_{U 2 V_{2}}=$ $\left\{R_{U}, H_{U}\right\}$ is returned. Then, instance $U_{i}$ terminates and record $\left\{\left(\mathrm{IDD}_{U}, E_{K_{U}^{\prime}}\left(r_{U 1}\right), E_{r_{U 1}}\left(\mathrm{PWD}_{U}\right), M_{U 1}\right),\left(\mathrm{ID}_{V}, X, Y, M_{U 2 V_{1}}\right)\right.$, $\left.\left(M_{S 1}, M_{S 2}, M_{S 3}, M_{S 4}\right), \quad\left(R_{V}, H_{V_{2}}, M_{S 1}, M_{S 2}\right),\left(R_{U}, H_{U}\right)\right\} \quad$ is added to list $L_{S}$.

Rule $V 5^{(5)}: R_{U V}^{\prime} \leftarrow r_{V_{2}} \cdot R_{U}, k_{U V}^{\prime} \leftarrow H_{2}\left(R_{U} \| R_{V}\right)$, and instance $V_{j}$ terminates.

Games $G M_{5}$ and $G M_{4}$ are almost indistinguishable only if the following event Ask $H 5$ occurs: $A$ queries the hash function $H_{2}(\cdot)$ on $\left(R_{U}\left\|R_{V}\right\| R_{U V}\right)$ or on $\left(R_{U}\left\|R_{V}\right\| R_{U V}^{\prime}\right)$. Because the $H_{4}(\cdot)$ is a private oracle, the probability that $A$ correctly guesses the value of $b$ is $1 / 2$. Hence,

$$
\begin{gathered}
\left|\operatorname{Pr}\left[\mathrm{Succ}_{5}\right]-\operatorname{Pr}\left[\mathrm{Succ}_{4}\right]\right| \leq \operatorname{Pr}[\text { AskH5 }], \\
\operatorname{Pr}\left[\mathrm{Succ}_{5}\right]=\frac{1}{2} .
\end{gathered}
$$

Game $G M_{6}$. In this game, the random self-reducibility of the $\mathrm{CDH}$ problem is used to simulate the executions. Given a $\mathrm{CDH}$ instance $(A, B)$, where $A, B \in G_{1}$, the next rules are listed.

Rule $V 3^{(6)}: a \leftarrow Z_{q}^{*}, r_{S 1}^{\prime} \leftarrow M_{S 1} \oplus k_{S V x}^{\prime} \oplus k_{S V y}^{\prime}, R_{V} \leftarrow$ $a \cdot A, H_{V_{2}} \leftarrow H_{2}\left(R_{V x}\left\|R_{V y}\right\| r_{S 1}^{\prime}\right)$. The message $M_{V_{2} U}=$ $\left\{R_{V}, H_{V_{2}}, M_{S 2}, M_{S 3}\right\}$ is returned. Then, instance $V_{j}$ proceeds to an expecting state.

Rule $U 4^{(6)}: b \leftarrow Z_{q}^{*}, R_{U} \leftarrow b \cdot B, H_{U} \leftarrow H_{2}\left(R_{U x}\left\|R_{U y}\right\| r_{S 1}^{\prime \prime}\right)$. Instance $U_{i}$ accepts and proceeds.

By the definition of event AskH5, event AskH6 means that the adversary has issued a query to random oracle on $\left(R_{U}\left\|R_{V}\right\| \mathrm{CDH}\left(R_{U}, R_{V}\right)\right)$. The number of records, such as $(2, *, *)$ in the list $L_{A}$, is $q_{2}$, and the probability of obtaining the $\operatorname{CDH}\left(R_{U}, R_{V}\right)$ value from list $L_{A}$ is $1 / q_{2}$. Hence, the accumulated probability is $\left(1 / q_{2}\right) \times \operatorname{Pr}[$ AskH6]. Besides, the $\mathrm{CDH}$ problem is random characteristics of the selfreducibility, and the equation $\operatorname{CDH}\left(R_{U}, R_{V}\right)=\operatorname{CDH}(A, B)^{a b}$ holds. Thus,

$$
\begin{gathered}
\operatorname{Pr}[\text { AskH5] }=\operatorname{Pr}[\text { AskH6], } \\
\operatorname{Pr}\left[\text { AskH6] }=q_{2} \operatorname{Succ}_{P, G_{1}}^{\mathrm{CDH}}\left(t^{\prime}\right) .\right.
\end{gathered}
$$

Finally, we summarize all the relationships and complete the proof.

From (50) to (53), we could derive

$$
\begin{aligned}
\left|\operatorname{Pr}\left[\mathrm{Succ}_{0}\right]-\operatorname{Pr}\left[\mathrm{Succ}_{4}\right]\right| \leq & \frac{q_{1}^{2}+q_{3}^{2}+q_{s s}+10 q_{2}+2 q_{3}}{2(q-1)} \\
& +\frac{q_{2}^{2}+2 q_{s s}+4 q_{s c}+4 q_{s v}}{2^{l+1}} .
\end{aligned}
$$

From (54) to (55), we obtain

$$
\left|\operatorname{Pr}\left[\mathrm{Succ}_{4}\right]-\frac{1}{2}\right| \leq q_{2} \operatorname{Succ}_{P, G_{1}}^{\mathrm{CDH}}\left(t^{\prime}\right) .
$$

From (56) and (57), we have

$$
\begin{aligned}
\left|\operatorname{Pr}\left[\operatorname{Succ}_{0}\right]-\frac{1}{2}\right| \leq & \frac{q_{1}^{2}+q_{3}^{2}+q_{s s}+10 q_{2}+2 q_{3}}{2(q-1)} \\
& +\frac{q_{2}^{2}+2 q_{s s}+4 q_{s c}+4 q_{s v}}{2^{l+1}} \\
& +q_{2} \operatorname{Succ}_{P, G_{1}}^{\mathrm{CDH}}\left(t^{\prime}\right) .
\end{aligned}
$$

From (49) and (58), we obtain (48) and the Theorem 1.

\section{Security Analysis of Our Scheme}

To get over the problems existing in Yang et al's scheme, we proposed a provable secure and efficient authentication scheme using smart card based on elliptic curve cryptography. In this part, we will show that the scheme we proposed is secure against various attacks $[23,24]$. 
7.1. Smart Card Loss Attack. The smart card of user $U$ contains $\left\{r_{U}, S_{U}, P_{U}, M_{U}, P, H_{2}(\cdot), E_{K}(\cdot) / D_{K}(\cdot)\right\}$. If the smart card of the user $U$ is stolen by the attacker, he/she could only get the secret data $r_{U}, S_{U}, P_{U}$, and $M_{U}$ from it; other data in the smart card is public to all clients. However, he/she does not know the identity $\mathrm{ID}_{U}$ or the password $\mathrm{PW}_{U}$ of $U$. As a result, he/she cannot use the secret data $r_{U}, S_{U}, P_{U}$, and $M_{U}$ to impersonate the user $U$ to pass the authentication of the server and the device. As the result, our scheme can resist the smart card loss attack.

7.2. Denial of Service Attack. In some schemes [10, 25-27], both of the server and user need to update some shared data in their smart card or verifier table after the key agreement phase or the authentication phase. The attacker can eavesdrop, intercept, and modify any transmitted messages on the public channel. And the behavior of the attacker may cause the difference of the shared data between the user and the server. So, these schemes cannot resist the denial of service attack. In our proposed scheme, the user and the server have not needed to update some data in their smart card or verifier table. Thus, the attacker no longer can perform the denial of service attack.

7.3. Efficient Login Phase. To improve the efficiency of our proposed scheme, before the key agreement phase, the smart card checks the correctness of user's identity and password. In the login phase of our scheme, when the user $U$ inputs his/her identity $\mathrm{ID}_{U}$ and password $\mathrm{PW}_{U}$, the smart card checks the correctness of $\mathrm{ID}_{U}$ and $\mathrm{PW}_{U}$ firstly through the equation $P_{U}=H_{2}\left(S_{U} \oplus H_{2}\left(\mathrm{PW}_{U} \oplus r_{U}\right) \oplus M_{U} \oplus \mathrm{ID}_{U}\right)$. If it does not hold, the smart card reject $U$ 's request. Otherwise, it authenticates the legality of the user $U$ and turns to the key agreement phase.

7.4. Session Key Attack. Firstly, the security of the session key in our scheme is based on the computational DiffieHellman problem. Secondly, the session key is generated by the random numbers, which are randomly selected by the user and the device, respectively. At last, before computing a session key, both of them must authenticate each other [28]. Based on the reasons mentioned above, the attacker cannot perform the session key attack.

7.5. Insider Attack. Because of without verifier table in this system, the insider cannot acquire any secret data from the server's system. In addition, the insider cannot obtain $K_{U}$ without the server's private key. And the adversary has no idea to derive the secret data $K_{U}$ from all messages he/she can achieve. Thus, he/she cannot impersonate a legal user $U^{\prime}$ to pass the authentication of the server and the device, or the server $S^{\prime}$ to deceive a legal user. Therefore, the proposed scheme is able to withstand the insider attack.

7.6. Replay Attack. If the U's message $M_{U 2 V_{1}}$ is intercepted and resent to the device by the attacker, the message $M_{V_{2} S}$ is computed and sent to the server by the device. Obviously the user and the device can through the server's certification, and the server responses the message $M_{S 2 V}$ to the device. After that, the device can authenticate the server and sends the message $M_{V_{2} U}$ to the attacker. The attacker can acquire nothing from $\mathrm{M}_{V_{2} U}$. So, he/she cannot send a legality message $M_{U 2 V_{2}}$ to the device. The device must not establish a session key with the attacker [29]. In the same way, if the attacker replays the device's message, he/she also cannot pass the user's authentication.

\section{Performance Comparisons}

In this part, our proposed scheme's performance will be evaluated compared with some other schemes [10, 12]. The comparison is summarized in Table 1. We define seven parameters of time complexity which are adopted in the schemes mentioned above as follows.

(i) $T_{H 1}$ : The time complexity of executing a hash function $H_{1}(\cdot)$.

(ii) $T_{H 2}$ : The time complexity of executing a hash function $\mathrm{H}_{2}(\cdot)$.

(iii) $T_{H_{3}}$ : The time complexity of executing a hash function $\mathrm{H}_{3}(\cdot)$.

(iv) $T_{P}$ : The time complexity of executing a pairings operation of point on elliptic curve.

(v) $T_{M}$ : The time complexity of executing a scalar multiplication operation of point on elliptic curve.

(vi) $T_{A}$ : The time complexity of executing an addition operation of point on elliptic curve.

(vii) $T_{S}$ : The time complexity of executing a symmetric key computation.

A comparison of our proposed scheme and that of Zhang et al. and Yang et al. is summarized in Table 1. It is known to all that one-way hash function is more efficient than the operation of scalar multiplication. Moreover, the pairing operation costs much more than the scalar multiplication operation. The effort of evaluating one pairing operation is approximately three times the effort of evaluating one scalar multiplication operation. Therefore our proposed scheme performs better than Zhang et al's scheme and Yang et al's scheme. Consequently, our proposed scheme is much more suitable for practical applications.

\section{Conclusions}

We have analyzed the scheme of Yang et al. and pointed out, except the attacks mentioned in Mishra et al. paper, their scheme suffers from the session key attack and has some mistakes. We propose a new provable secure and efficient digital rights management authentication scheme using smart card based on elliptic curve cryptography to surmount the problems in Yang et al.s. And we demonstrate that the new scheme is provable secure under the model AFP05 introduced in this paper. Because hash function is used to replace the operations of point on elliptic curve and the symmetric key computation in our scheme, our scheme 
TABLE 1: Performance comparisons.

\begin{tabular}{|c|c|c|c|}
\hline & Our proposed scheme & Yang et al.s scheme & Zhang et al's scheme \\
\hline \multicolumn{4}{|c|}{$\begin{array}{l}\text { Registration } \\
\text { phase }\end{array}$} \\
\hline$U$ & $2 T_{H 2}$ & $1 T_{H 2}$ & - \\
\hline$S$ & $3 T_{H 1}+3 T_{H 2}+3 T_{M}$ & $2 T_{H 1}+1 T_{H 2}+2 T_{M}$ & $2 T_{H 1}+4 T_{H 2}+2 T_{M}$ \\
\hline \multicolumn{4}{|c|}{$\begin{array}{l}\text { Login phase and } \\
\text { key agreement } \\
\text { phase }\end{array}$} \\
\hline$U$ & $9 T_{H 2}+2 T_{M}+2 T_{S}$ & $6 T_{H 2}+6 T_{S}$ & $7 T_{H 2}+7 T_{S}$ \\
\hline$V$ & $4 T_{H 2}+1 T_{H 3}+6 T_{M}+1 T_{A}$ & $2 T_{H 2}+2 T_{H 3}+3 T_{P}+6 T_{M}+2 T_{A}+3 T_{S}$ & $3 T_{H 2}+2 T_{H 3}+3 T_{P}+6 T_{M}+2 T_{A}+3 T_{S}$ \\
\hline$S$ & $\begin{array}{c}3 T_{H 1}+6 T_{H 2}+1 T_{H 3}+1 T_{P}+5 T_{M}+ \\
1 T_{A}+2 T_{S}\end{array}$ & $\begin{array}{c}1 T_{H 1}+5 T_{H 2}+2 T_{H 3}+2 T_{P}+7 T_{M}+ \\
3 T_{A}+5 T_{S}\end{array}$ & $5 T_{H 2}+2 T_{H 3}+2 T_{P}+7 T_{M}+3 T_{A}+6 T_{S}$ \\
\hline \multicolumn{4}{|c|}{$\begin{array}{l}\text { Password } \\
\text { update phase }\end{array}$} \\
\hline$U$ & $9 T_{H 2}+2 T_{S}$ & $4 T_{H 2}$ & - \\
\hline$S$ & $3 T_{H 1}+7 T_{H 2}+3 T_{M}+2 T_{S}$ & $1 T_{H 1}+3 T_{H 2}$ & $4 T_{H 2}$ \\
\hline Total & $\begin{array}{c}9 T_{H 1}+40 T_{H 2}+2 T_{H 3}+1 T_{P}+ \\
19 T_{M}+2 T_{A}+4 T_{S} \\
\end{array}$ & $\begin{array}{c}4 T_{H 1}+22 T_{H 2}+4 T_{H 3}+5 T_{P}+ \\
15 T_{M}+5 T_{A}+8 T_{S} \\
\end{array}$ & $\begin{array}{c}2 T_{H 1}+23 T_{H 2}+4 T_{H 3}+5 T_{P}+ \\
15 T_{M}+5 T_{A}+9 T_{S} \\
\end{array}$ \\
\hline
\end{tabular}

is more efficient than Yang et al's scheme. As a result, our proposed scheme is more suitable for practical applications in ubiquitous computing.

\section{Notations}

$\begin{array}{ll}U: & \text { The user } \\ S: & \text { The server } \\ V: & \text { The device } \\ A: & \text { The attacker } \\ \mathrm{ID}_{U}: & \text { The user } U^{\prime} \text { ' identity } \\ s: & \text { The secret key of the server } S \\ P_{S}: & \text { The public key of the server } S \text { and } \\ & P_{S}=s P \\ \mathrm{PW}_{U}: & \text { The user } U^{\prime} \text { password } \\ P_{U}: & \text { The public key of the user } U \text { and } \\ & P_{U}=s H_{1}(\text { ID } U \\ H_{1}(\cdot): & \{0,1\}^{*} \mapsto G_{1}, \text { a one-way hash } \\ & \text { function maps an arbitrary length } \\ & \text { bit string into a member of group } \\ & G_{1} \cdot \\ H_{2}(\cdot): & \{0,1\}^{*} \mapsto\{0,1\}^{l}, \text { a one-way hash } \\ & \text { function maps an arbitrary length } \\ & \text { bit string into a } l \text {-bits string. } \\ & \{0,1\}^{*} \mapsto Z_{q}^{*}, \text { a one-way hash } \\ H_{3}(\cdot): & \text { function maps an arbitrary length } \\ & \text { bit string into a random member } \\ & \text { in group } Z_{q}^{*} \cdot \\ E_{K}(\cdot) / D_{K}(\cdot): & \text { The symmetric } \\ & \text { encryption/decryption algorithm } \\ & \text { using key } K \\ & \text { The bitwise XOR operation } \\ & \text { String concatenation operation. } \\ & \end{array}$

\section{Conflict of Interests}

The authors declare that there is no conflict of interests regarding the publication of this paper.

\section{Acknowledgment}

The authors would like to extend their sincere appreciation to the Deanship of Scientific Research at King Saud University for its funding of this research through the Research Group Project no. RGP-VPP-288.

\section{References}

[1] M. Weiser, "The computer for the 21st century," Scientific American, pp. 94-104, 1991, reprinted in IEEE Pervasive Computing, 2002: 19-25.

[2] B. Alomair and R. Poovendran, "Efficient authentication for mobile and pervasive computing," in Information and Communications Security, vol. 6476 of Lecture Notes in Computer Science, pp. 186-202, Springer, Berlin, Germany, 2010.

[3] D. H. Seo and I. Y. Lee, "A study on RFID system with secure service availability for ubiquitous computing," Journal of Information Processing Systems, vol. 1, no. 1, pp. 96-101, 2005.

[4] T. M. Thanh and M. Iwakiri, "A proposal of digital rights management based on incomplete cryptography using invariant Huffman code length feature," Multimedia Systems, vol. 20, no. 2, pp. 127-142, 2014.

[5] S. W. Park and I. Y. Lee, "Anonymous authentication scheme based on NTRU for the protection of payment information in NFC mobile environment," Journal of Information Processing Systems, vol. 9, no. 3, pp. 461-476, 2013.

[6] D. Kirovski, M. Peinado, and F. A. P. Petitcolas, "Digital rights management for digital cinema," in Proceedings of the International Society for Optical Engineering, pp. 105-120, August 2001. 
[7] S. Emmanuel and M. S. Kankanhalli, "A digital rights management scheme for broadcast video," Multimedia Systems, vol. 8, no. 6, pp. 444-458, 2003.

[8] H. Chang and M. J. Atallah, "Protecting software code by guards," in DRM: ACM CCS-8 Workshop on Security and Privacy in Digital Rights Management, pp. 160-175, Springer, Berlin, Germany, 2002.

[9] A. Seki and W. Kameyama, "A proposal on open DRM system coping with both benefits of rights-holders and users," in Proceedings of the IEEE Global Telecommunications Conference (GLOBECOM '03), vol. 7, pp. 4111-4115, December 2003.

[10] H. W. Yang, C. C. Yang, and W. Lin, "Enhanced digital rights management authentication scheme based on smart card," IET Information Security, vol. 7, no. 3, pp. 189-194, 2013.

[11] U. J. Jang, H. Lim, and Y. Shin, "A license audit model for secure DRM systems in IP-based environments," Journal of Information Processing Systems, vol. 6, no. 2, pp. 253-260, 2010.

[12] Y. C. Zhang, L. Yang, P. Xu, and Y. S. Zhan, "A DRM authentication scheme based on smart-card," in Proceedings of the International Conference on Computational Intelligence and Security (CIS '09), pp. 202-207, Beijing, China, December 2009.

[13] D. Mishra and S. Mukhopadhyay, "Cryptanalysis of Yang et al.s digital rights management authentication scheme based on smart card," in Recent Trends in Computer Networks and Distributed Systems Security, vol. 420 of Communications in Computer and Information Science, pp. 288-297, 2014.

[14] C. C. Lee, M. S. Hwang, and W. P. Yang, "A flexible remote user authentication scheme using smart cards," ACM Operating Systems Review, vol. 36, no. 3, pp. 45-52, 2002.

[15] J. J. Yuan, "An enhanced two-factor user authentication in wireless sensor networks," Telecommunication Systems, vol. 55, no. 1, pp. 105-113, 2014.

[16] J. Petit and Z. Mammeri, "Authentication and consensus overhead in vehicular ad hoc networks," Telecommunication Systems, vol. 52, no. 4, pp. 2699-2712, 2013.

[17] H. M. Yang, Y. X. Zhang, and Y. Z. Zhou, "Provably secure threeparty authenticated key agreement protocol using smart cards," Computer Networks, vol. 58, pp. 29-38, 2014.

[18] M. Abdalla, P. A. Fouque, and D. Pointcheval, "Password based authenticated key exchange in the three-party setting," in Proceedings of the International Workshop on Practice and Theory in Public Key Cryptography (PKC '05), pp. 65-84, 2005.

[19] C. Tsai, C. Lee, and M. Hwang, "Password authentication schemes: current status and key issues," International Journal of Network Security, vol. 3, no. 2, pp. 101-115, 2006.

[20] J. W. K. Gnanaraj, K. Ezra, and E. B. Rajsingh, "Smart card based time efficient authentication scheme for global grid computing," Human-Centric Computing and Information Sciences, vol. 3, no. 1, pp. 1-14, 2013.

[21] X. Wang, W. Guo, W. Zhang, M. K. Khan, and K. Alghathbar, "Cryptanalysis and improvement on a parallel keyed hash function based on chaotic neural network," Telecommunication Systems, vol. 52, no. 2, pp. 515-524, 2013.

[22] J. Katz and Y. Lindell, Introduction to Modern Cryptography, Chapman \& Hall/CRC Press, 2007.

[23] C. Lee, T. Lin, and R. Chang, "A secure dynamic ID based remote user authentication scheme for multi-server environment using smart cards," Expert Systems with Applications, vol. 38, no. 11, pp. 13863-13870, 2011.

[24] C. Lee, C. Chen, P. Wu, and T. Chen, "Three-factor control protocol based on elliptic curve cryptosystem for universal serial bus mass storage devices," IET Computers \& Digital Techniques, vol. 7, no. 1, pp. 48-55, 2013.

[25] L. Cao and W. Ge, "Analysis and improvement of a multi-factor biometric authentication scheme," Security and Communication Networks, 2014.

[26] Y. An, "Security analysis and enhancements of an effective biometric-based remote user authentication scheme using smart cards," Journal of Biomedicine and Biotechnology, vol. 2012, Article ID 519723, 6 pages, 2012.

[27] M. K. Khan and J. Zhang, "An efficient and practical fingerprintbased remote user authentication scheme with smart cards," in Information Security Practice and Experience, pp. 260-268, Springer, Berlin, Germany, 2006.

[28] T.-T. Truong, M.-T. Tran, and A.-D. Duong, "Improvement of the more efficient \& secure ID-based remote mutual authentication with key agreement scheme for mobile devices on ECC," Journal of Convergence, vol. 3, no. 2, pp. 19-30, 2012.

[29] Y. Chung, S. Choi, and D. Won, "Lightweight anonymous authentication scheme with unlinkability in global mobility networks," Journal of Convergence, vol. 4, no. 4, pp. 23-29, 2013. 


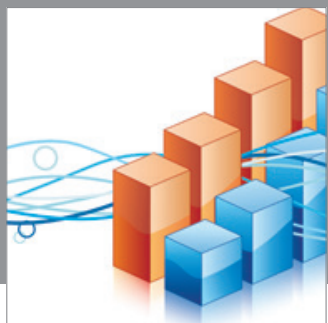

Advances in

Operations Research

mansans

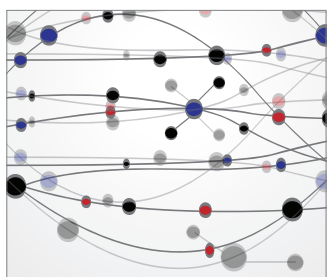

The Scientific World Journal
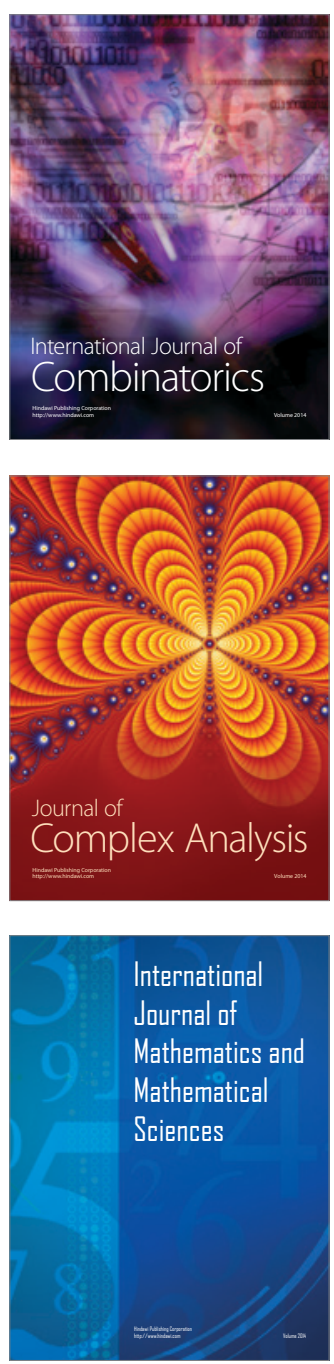
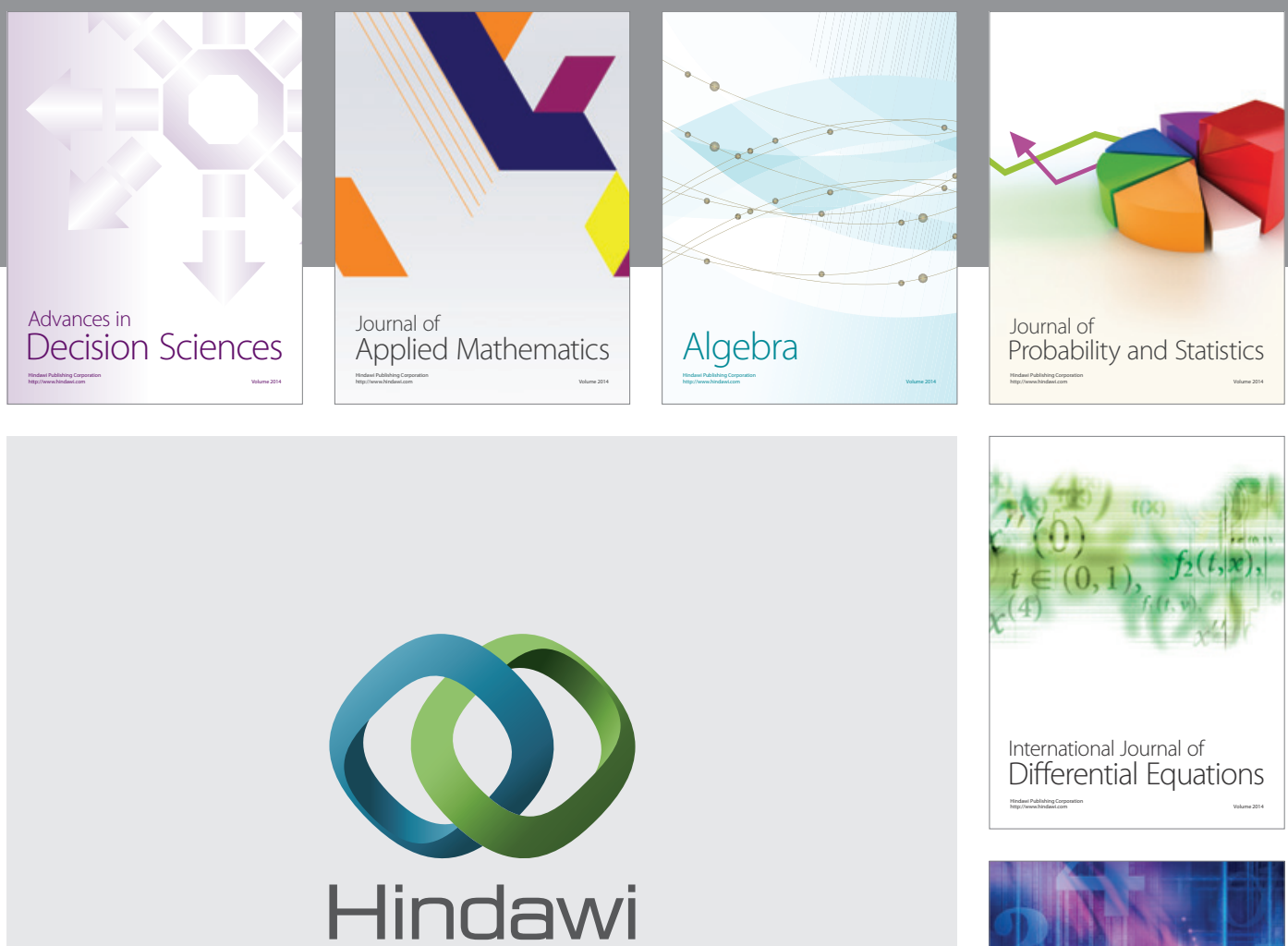

Submit your manuscripts at http://www.hindawi.com
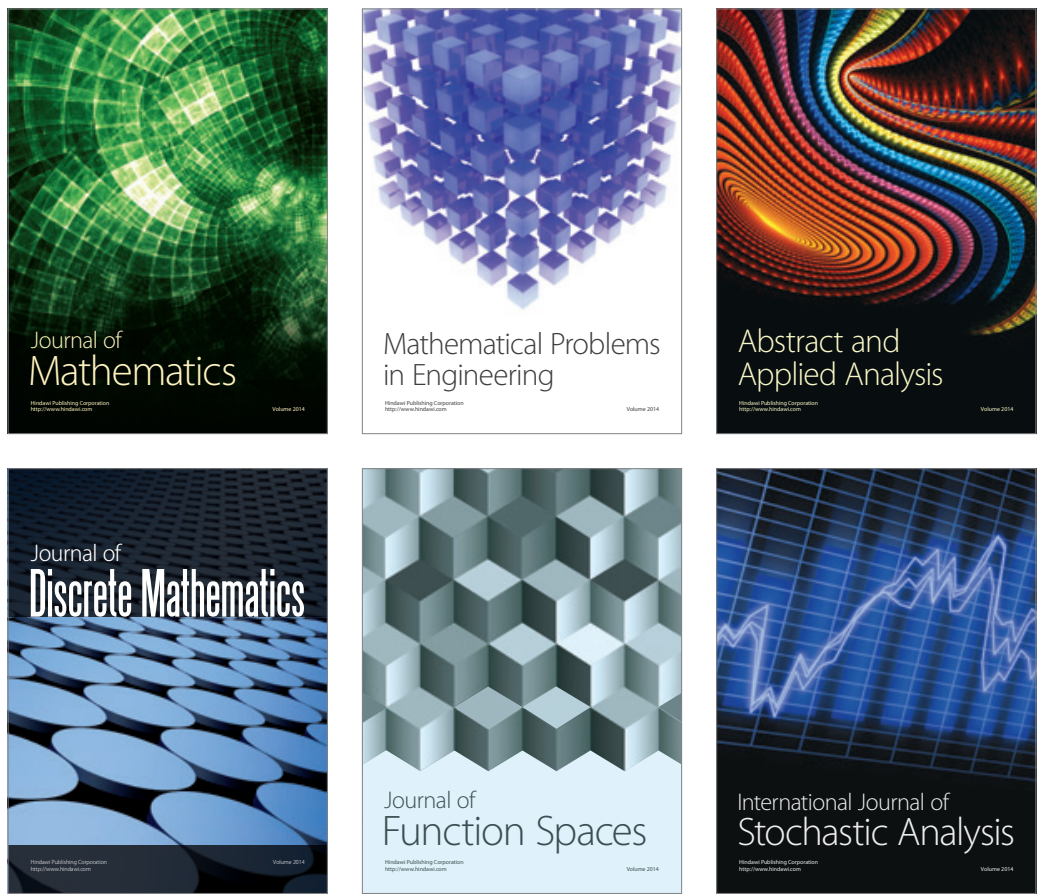

Journal of

Function Spaces

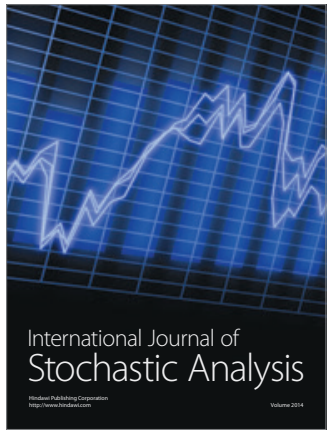

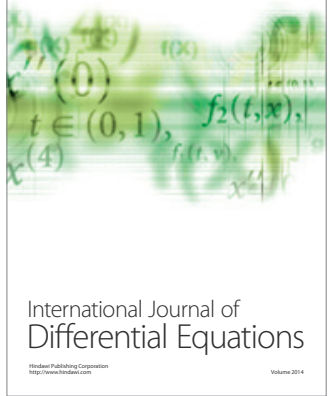
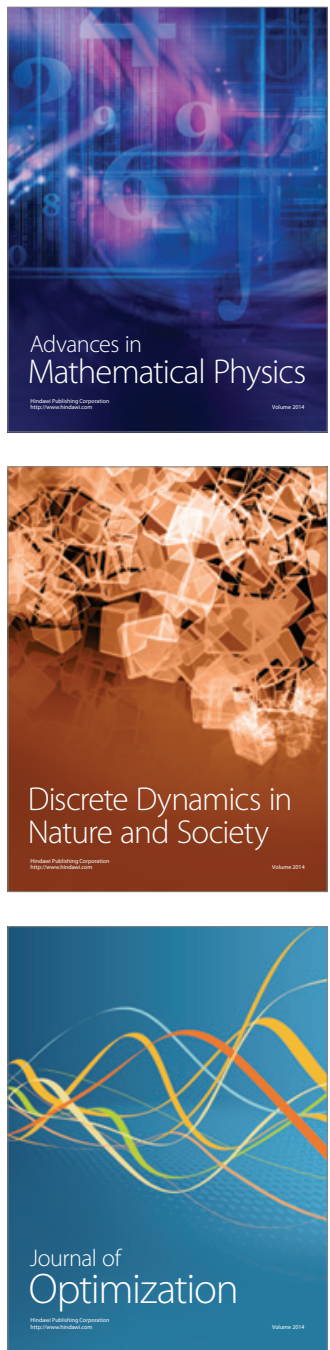